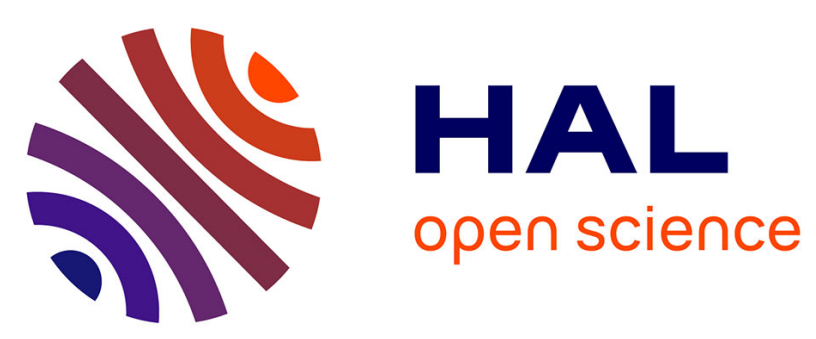

\title{
Solar and Heliospheric Observatory/solar Wind Anisotropies Observations of five moderately bright comets: 1999-2002
}

\author{
Michael R. Combi, J. Teemu T. Mäkinen, N.J. Henry, Jean-Loup Bertaux, \\ Eric Quémerais
}

\section{To cite this version:}

Michael R. Combi, J. Teemu T. Mäkinen, N.J. Henry, Jean-Loup Bertaux, Eric Quémerais. Solar and Heliospheric Observatory/solar Wind Anisotropies Observations of five moderately bright comets: 1999-2002. The Astronomical Journal, 2008, 135 (4), pp.1533-1550. 10.1088/0004-6256/135/4/1533 . hal-00269407

\section{HAL Id: hal-00269407 https://hal.science/hal-00269407}

Submitted on 18 Jul 2020

HAL is a multi-disciplinary open access archive for the deposit and dissemination of scientific research documents, whether they are published or not. The documents may come from teaching and research institutions in France or abroad, or from public or private research centers.
L'archive ouverte pluridisciplinaire HAL, est destinée au dépôt et à la diffusion de documents scientifiques de niveau recherche, publiés ou non, émanant des établissements d'enseignement et de recherche français ou étrangers, des laboratoires publics ou privés. 


\title{
SOLAR AND HELIOSPHERIC OBSERVATORY/SOLAR WIND ANISOTROPIES OBSERVATIONS OF FIVE MODERATELY BRIGHT COMETS: 1999-2002
}

\author{
M. R. Combi ${ }^{1}$, J. T. T. MäKInen ${ }^{2}$, N. J. Henry ${ }^{1}$, J.-L. BertauX ${ }^{3}$, And E. Quemérais ${ }^{3}$ \\ ${ }^{1}$ Department of Atmospheric, Oceanic and Space Sciences, University of Michigan, 2455 Hayward Street, Ann Arbor, MI 48109-2143, USA; mcombi@umich.edu \\ 2 Finnish Meteorological Institute, Box 503, SF-00101 Helsinki, Finland \\ ${ }^{3}$ Service d'Aéronomie du CNRS, Université de Versailles Saint-Quentin, BP3, 91371 Verrières le Buisson Cedex, France \\ Received 2007 November 28; accepted 2008 February 5; published 2008 March 12
}

\begin{abstract}
Solar Wind Anisotropies (SWAN), the all-sky hydrogen Lyman-alpha camera, on the SOHO spacecraft makes routine all-sky images of the interplanetary neutral hydrogen around the Sun and thus monitors the effect of the variable solar wind on its distribution. SWAN has an ongoing campaign to make special observations of comets, both short- and long-period ones, in addition to making serendipitous observations of comets as part of the all-sky monitoring program. We report here on a study of the moderately active Oort cloud comets observed by SWAN during the period of 1999-2002: 1999 H1 Lee, 1999 T1 McNaught Hartley, 2000 WM1 LINEAR, 2001 A2 LINEAR, and 2002 C1 Ikeya Zhang (P153). SWAN is able to observe comets almost continuously over most of their visible apparitions and provide excellent temporal coverage of water production. In addition to calculating production rates from each single image, we also present results using our time-resolved model (TRM) that analyzes an entire sequence of images over many days to several weeks/months, and from which daily-averaged or two-dayaveraged water production rates are extracted over continuous periods of several days to months. The short-term (outburst) behavior is correlated with other observations and is examined and associated with fragment release. The long-term heliocentric distance-dependent variations of water production rate are examined and compared and contrasted with the measured volatile compositions of the comets as well as their absolute production rate levels. The overall long-term variation is also distinguished from seasonal effects seen in the pre- to post-perihelion differences.
\end{abstract}

Key words: comets: general - comets: individual (1999 H1 Lee, 1999 T1 McNaught-Hartley, 2001 A2 LINEAR, 2001 WM1 LINEAR, P153/Ikeya-Zhang) - molecular processes

\section{INTRODUCTION}

Observations of hydrogen Lyman- $\alpha(\operatorname{Ly}-\alpha)$ at $1215.7 \AA$ in comets and their interpretation are important. Atomic hydrogen is the most abundant species in the atmosphere (or coma) of a comet being produced in a photodissociation chain originating with water molecules and including intermediate $\mathrm{OH}$ radicals. Water is the most abundant volatile species in a comet's nucleus, and water sublimation controls the abundance and activity of the coma when comets are within $3 \mathrm{AU}$ from the Sun. Measurements of the abundance and distribution of hydrogen in the coma, when appropriately modeled, can provide a reliable measure of the water production rate and its variation in time in comets. Virtually all compositional information is compared to water, making water the most important species for obtaining accurate production rates. Variations in production rate with time generally, and with heliocentric distance in particular can provide information about the composition and structure of the nucleus.

Solar Wind Anisotropies (SWAN), the all-sky hydrogen Ly- $\alpha$ camera, has been operating on the Solar and Heliospheric Observatory $(\mathrm{SOHO})$ spacecraft since its launch in 1995. The SWAN instrument was designed to observe the entire sky in $\mathrm{H}$ Ly- $\alpha$ in order to obtain a global view of the variable interaction of the solar wind with the neutral interstellar hydrogen streaming through the solar system. From its viewpoint at the L1 Lagrange point between the Earth and Sun it obtains an unparallel view of the Sun, its large extended corona, and the entire sky. For a more detailed description of SWAN, see Bertaux et al. (1995).

Because of the limited observing time available for synoptic cometary observations (or any solar system targets for that matter) with the Hubble Space Telescope (HST), compounded by the fact that many bright comets are often within the solar avoidance area of the HST, space-based observations, and in particular ultraviolet (UV) observations, have been severely limited in recent years compared with most of the previous 20 years when IUE was available. SWAN with its UV capability has filled an important void in the monitoring of comets from space since early 1996.

Because of the large neutral hydrogen coma, SWAN can observe comets either in the nominal full-sky mode, when comets are recorded during the nominal full-sky interplanetary medium observations, or during special campaigns of cometspecific observations, when the region of the sky with a comet is specifically targeted and sometimes oversampled by the SWAN instrument field of view (IFOV) to yield a somewhat improved spatial resolution. We report here on a set of moderately bright comets observed by SWAN during the period of 1999-2004, which includes 1999 H1 Lee, 1999 T1 McNaught Hartley, 2000 WM1 LINEAR, 2001 A2 LINEAR, and 2002 C1 Ikeya Zhang (P153). The single-image water production rates are presented. We also used our time-resolved model (TRM) to analyze long sequences of images simultaneously to obtain deconvolved daily-average or two-day-average water production rates between the image snapshots. Because we are presenting results from several comets, the paper is organized such that we discuss comet observations generally, followed by a discussion of the model analysis procedure, and then followed by a discussion of each comet. In each of these sections, we examine the variation of the water production rates with time and heliocentric distance, discuss their significance, and compare with other observations. The paper concludes with a general summary. 


\section{OBSERVATIONS}

The observations presented in this paper result from two basic observational modes. SWAN routinely observes the full sky two to three times per week. Any comet bright enough will be registered on full-sky images. When a bright or otherwise interesting comet is expected, comet-specific observations are also planned and made. The comet-specific images can improve the spatial resolution by oversampling and reduce the noise by longer integrations over the full-sky images (Mäkinen \& Combi 2005). For the previous results on comet 1996 B2 (Hyakutake) the comet-specific images were more important when the comet was very close to the Earth because of its large proper motion during the time the full-sky images are acquired. In that case Combi et al. (2005) accounted for any motion issues in the daily-averaged deconvolution with the TRM. These effects are included in the formal uncertainties in the single-image production rates and as well in the daily-averaged deconvolved results given in the next section. Tables giving the observational circumstances for all the SWAN images for each comet as well as the single-image production rates are presented below in the sections on each comet. The $g$-factor was derived from dailyaverage observations by the SOLSTICE instrument on UARS, which measures the solar UV irradiance. It is corrected to first order for the solar radiation difference between the face of the Sun seen by $\mathrm{SOHO}$ and that seen by the comet (Combi et al. 2000) and for the Doppler shift of the Ly- $\alpha$ line profile caused by the heliocentric radial velocity of the comet.

\section{TIME-RESOLVED MODEL}

We have used the TRM to extract the time history of the water production rate by analyzing the various series of images together. The details of the model are described in the paper by Mäkinen \& Combi (2005). Because of its long lifetime ( $1.5 \times$ $10^{6} \mathrm{~s}$ at $1 \mathrm{AU}$ from the Sun), $\mathrm{H}$ atoms survive in the coma for $2-4$ weeks, during which time there are often available up to five to ten SWAN images of the coma. The TRM uses a parametrized velocity distribution for $\mathrm{H}$ atoms in the coma, estimated from explicit calculations of the partial thermalization by the heavyatom coma (Combi \& Smyth 1988a, 1988b; Combi et al. 2000), and tracks their propagation out into the coma. The method then builds up a set of basis functions relating the production rate of water at the nucleus as a function of time to the distribution of photodissociated $\mathrm{H}$ atoms as seen on the sky plane. In this paper, we present results of the water production rates in the standard single-image form as well as daily-averaged and twoday-averaged deconvolved water production rates extracted by the inversion scheme of the TRM.

\section{1999 H1 LEE}

Comet 1999 H1 Lee (hereafter also referred to as Lee) is a long-period Oort cloud comet that reached a perihelion distance of $0.708 \mathrm{AU}$ on 1999 July 11.1772 UT. Observations of the composition of Lee generally found abundances of most volatiles to be comparable to Oort cloud comets, except for $\mathrm{CO}$, which was only present at the 2-4\% level (Biver et al. 2000; Mumma et al. 2001b). SWAN observations show the $\mathrm{H}$ coma of comet Lee from 1999 April 10 to September 20 covering a heliocentric distance range from $1.43 \mathrm{AU}$ before perihelion to 1.48 after. Table 1 gives the observational circumstances and single-image water production rates determined using the TRM. In this mode an entire image is analyzed, assuming a nominal time/heliocentric distance variation $\left(r^{-2}\right)$, and a single production rate is determined for the time of the observation using the entire image. The top panel of Figure 1 shows the pre- and post-perihelion production rates plotted as a function of heliocentric distance. Past experience shows that the long-term heliocentric distance dependence, usually fitted as a variation in the usual $r^{-p}$ form, is well represented in this way, as are general pre- to post- perihelion variation asymmetries. The daily-averaged deconvolution is most suited to fill in gaps between images and for correct time phasing of transient short-term activity variations. The powerlaw fits are shown as straight lines in the top set of panels in Figure 1. The power-law fits for comet Lee are given in the top line of Table 2 for the pre- and post-perihelion legs as well as the averaged pre- and post-perihelion values.

Chiu et al. (2001) observed Lee with the Submillimeter Wave Astronomy Satellite (SWAS) and determined water production rates over the range of heliocentric distances from 1.3 to 1.7 $\mathrm{AU}$ after perihelion. Over this range they reported a value of $1.45 \times 10^{29} \mathrm{~s}^{-1}$ at $1 \mathrm{AU}$ and an exponent of -5.5 . The SWAN observations on the other hand cover both legs but not as far out as 1.7 AU. Neufeld et al. (2000), on the other hand, presented a single water production rate from SWAS observations before perihelion on May 19-23.

Biver et al. (2000) reported on radio observations of various species in Lee with a number of radio telescopes. From observations of $\mathrm{OH}$ and assuming that the water production rate was 1.1 times the $\mathrm{OH}$ production rate they report a power law of 8.0 $\times 10^{28} r^{-2.3}$ water molecules $\mathrm{s}^{-1}$. Their firm results are limited to the pre-perihelion leg with a range of heliocentric distances from 1.36 to $0.93 \mathrm{AU}$. For the post-perihelion period when they had no good $\mathrm{OH}$ observations they monitored the gas production variation with heliocentric distance by following the production rate of $\mathrm{HCN}$, which they find is about $1 / 900$ of the water production rate. After perihelion they find that the gas production rate variation is steeper with an exponent closer to -3 , and in general agreement with the pre- to post-perihelion asymmetry we find. They also report that post-perihelion production rates are about $30 \%$ lower than pre-perihelion, where we find a ratio of the $1 \mathrm{AU}$ values of about $121 \%$ - however, the exact value of the ratio depends in part on the specific heliocentric distances covered and ratioed.

Other water production rates covering limited ranges of heliocentric distance have been reported by Weaver et al. (2002), Mumma et al. (2001b), Neufeld et al. (2000), Feldman et al. (1999), Lara et al. (2004a), and Dello Russo et al. (2005). These, as well as those discussed above from Chiu et al. and Biver et al., are plotted in Figure 2 along with the TRM dailyaveraged deconvolved water production rates from SWAN, which are given in Table 3. The values from Biver et al. show the same temporal variation but are about $25 \%$ below SWAN. The values of Chiu et al., as mentioned, are mostly at larger post-perihelion heliocentric distances, but are about half of the SWAN values in the overlap region. The values of Dello Russo et al., Mumma et al., Feldman et al., and Neufeld et al. are comparable with some a little larger and some a little smaller. The preliminary value of Weaver et al. (1999), as reported in Biver et al. (2000), from ground-based IR observations seems anomalously low compared with all other observations including IR observations by Dello Russo et al. and Mumma et al. Taken together, and acknowledging the usual systematic differences between different observed species and model parameters, there is general consistency in describing the higher pre-perihelion level and the steeper fall-off 
Table 1

Comet Lee: Observational Circumstances and Single-Image Water Production Rates

\begin{tabular}{|c|c|c|c|c|c|}
\hline$\Delta T$ (days) & $r(\mathrm{AU})$ & $\Delta(\mathrm{AU})$ & $g\left(\mathrm{~s}^{-1}\right)$ & $Q\left(\mathrm{~s}^{-1}\right)$ & $\Delta Q\left(\mathrm{~s}^{-1}\right)$ \\
\hline-66.544 & 1.434 & 0.722 & 0.002248 & $8.854 \times 10^{28}$ & $1.171 \times 10^{25}$ \\
\hline-65.374 & 1.417 & 0.723 & 0.002249 & $9.148 \times 10^{28}$ & $6.154 \times 10^{25}$ \\
\hline-63.979 & 1.397 & 0.727 & 0.002251 & $8.728 \times 10^{28}$ & $1.151 \times 10^{25}$ \\
\hline-63.472 & 1.389 & 0.729 & 0.002251 & $1.039 \times 10^{29}$ & $4.901 \times 10^{25}$ \\
\hline-61.516 & 1.361 & 0.740 & 0.002252 & $1.240 \times 10^{29}$ & $1.415 \times 10^{25}$ \\
\hline-60.468 & 1.346 & 0.748 & 0.002226 & $1.059 \times 10^{29}$ & $5.051 \times 10^{25}$ \\
\hline-59.502 & 1.332 & 0.757 & 0.002227 & $9.492 \times 10^{28}$ & $1.150 \times 10^{25}$ \\
\hline-58.412 & 1.316 & 0.767 & 0.002228 & $8.537 \times 10^{28}$ & $6.512 \times 10^{25}$ \\
\hline-56.504 & 1.289 & 0.789 & 0.002230 & $8.103 \times 10^{28}$ & $7.264 \times 10^{25}$ \\
\hline-52.316 & 1.229 & 0.848 & 0.002233 & $8.655 \times 10^{28}$ & $7.266 \times 10^{25}$ \\
\hline-50.724 & 1.206 & 0.874 & 0.002235 & $9.029 \times 10^{28}$ & $6.855 \times 10^{25}$ \\
\hline-49.660 & 1.191 & 0.892 & 0.002236 & $9.138 \times 10^{28}$ & $8.167 \times 10^{25}$ \\
\hline-46.665 & 1.149 & 0.946 & 0.002239 & $1.320 \times 10^{29}$ & $6.569 \times 10^{25}$ \\
\hline-44.606 & 1.120 & 0.985 & 0.002240 & $1.219 \times 10^{29}$ & $7.751 \times 10^{25}$ \\
\hline-42.679 & 1.093 & 1.022 & 0.002215 & $1.582 \times 10^{29}$ & $6.832 \times 10^{25}$ \\
\hline-37.608 & 1.024 & 1.123 & 0.002219 & $1.655 \times 10^{29}$ & $7.534 \times 10^{25}$ \\
\hline-35.692 & 0.999 & 1.161 & 0.002197 & $1.770 \times 10^{29}$ & $6.309 \times 10^{25}$ \\
\hline-32.695 & 0.960 & 1.221 & 0.002200 & $1.855 \times 10^{29}$ & $6.770 \times 10^{25}$ \\
\hline-30.637 & 0.934 & 1.261 & 0.002179 & $1.587 \times 10^{29}$ & $7.821 \times 10^{25}$ \\
\hline-28.991 & 0.914 & 1.293 & 0.002158 & $1.615 \times 10^{29}$ & $1.815 \times 10^{25}$ \\
\hline-28.709 & 0.911 & 1.298 & 0.002158 & $1.531 \times 10^{29}$ & $8.698 \times 10^{25}$ \\
\hline-25.380 & 0.872 & 1.360 & 0.002141 & $2.039 \times 10^{29}$ & $6.497 \times 10^{25}$ \\
\hline-22.994 & 0.846 & 1.403 & 0.002123 & $2.110 \times 10^{29}$ & $6.306 \times 10^{25}$ \\
\hline-19.175 & 0.807 & 1.467 & 0.002089 & $2.941 \times 10^{29}$ & $5.294 \times 10^{25}$ \\
\hline-16.905 & 0.786 & 1.502 & 0.002056 & $3.616 \times 10^{29}$ & $5.140 \times 10^{25}$ \\
\hline-14.648 & 0.768 & 1.5 & 0.002042 & $3.550 \times 10^{29}$ & $5.965 \times 10^{25}$ \\
\hline 10.193 & 0.738 & 1.682 & 0.001953 & $4.977 \times 10^{29}$ & $2.377 \times 10^{25}$ \\
\hline 14.101 & 0.764 & 1.666 & 0.001993 & $3.558 \times 10^{29}$ & $9.490 \times 10^{25}$ \\
\hline 15.771 & 0.777 & 1.657 & 0.0020 & $3.380 \times 10^{29}$ & $1.705 \times 10^{25}$ \\
\hline 25.544 & 0.874 & 1.569 & 0.002080 & $2.168 \times 10^{29}$ & $7.775 \times 10^{25}$ \\
\hline 27.479 & 0.896 & 1.546 & 0.002096 & $1.842 \times 10^{29}$ & $7.330 \times 10^{25}$ \\
\hline 30.493 & 0.932 & 1.507 & 0.002116 & $1.805 \times 10^{29}$ & $7.095 \times 10^{25}$ \\
\hline 32.563 & 0.958 & & & $1.749 \times 10^{29}$ & $7.274 \times 10^{25}$ \\
\hline 34.516 & 0.983 & 1.451 & 0.002136 & $1.375 \times 10^{29}$ & $8.404 \times 10^{25}$ \\
\hline 37.529 & 1.023 & 1.405 & 0.002156 & $1.120 \times 10^{29}$ & $8.214 \times 10^{25}$ \\
\hline 39.599 & 1.051 & 1.373 & 0.002158 & $8.701 \times 10^{28}$ & $9.015 \times 10^{25}$ \\
\hline 41.537 & 1.077 & 1.342 & 0.002159 & $9.014 \times 10^{28}$ & $8.464 \times 10^{25}$ \\
\hline 44.551 & 1.119 & 1.292 & 0.002181 & $8.433 \times 10^{28}$ & $8.805 \times 10^{25}$ \\
\hline 46.621 & 1.148 & 1.257 & 0.002182 & $8.324 \times 10^{28}$ & $9.218 \times 10^{25}$ \\
\hline 48.571 & 1.176 & 1.223 & 0.002184 & $9.669 \times 10^{28}$ & $7.807 \times 10^{25}$ \\
\hline 50.126 & 1.198 & 1.197 & 0.002185 & $9.035 \times 10^{28}$ & $1.554 \times 10^{25}$ \\
\hline 51.570 & 1.218 & 1.172 & 0.002186 & $8.852 \times 10^{28}$ & $7.435 \times 10^{25}$ \\
\hline 53.639 & 1.248 & 1.137 & 0.002187 & $6.985 \times 10^{28}$ & $8.800 \times 10^{25}$ \\
\hline 55.435 & 1.273 & 1.107 & 0.002189 & $6.484 \times 10^{28}$ & $8.996 \times 10^{25}$ \\
\hline 58.884 & 1.323 & 1.050 & 0.002191 & $4.787 \times 10^{28}$ & $1.086 \times 10^{26}$ \\
\hline 60.775 & 1.350 & 1.020 & 0.002192 & $5.152 \times 10^{28}$ & $1.048 \times 10^{26}$ \\
\hline 62.698 & 1.378 & 0.991 & 0.002213 & $5.098 \times 10^{28}$ & $1.133 \times 10^{26}$ \\
\hline 65.740 & 1.422 & 0.948 & 0.002215 & $3.883 \times 10^{28}$ & $1.185 \times 10^{26}$ \\
\hline 67.998 & 1.455 & 0.919 & 0.002217 & $4.078 \times 10^{28}$ & $1.026 \times 10^{26}$ \\
\hline 69.933 & 1.483 & 0.897 & 0.002199 & $3.719 \times 10^{28}$ & $1.099 \times 10^{26}$ \\
\hline
\end{tabular}

Notes.

$\Delta T$ : time from perihelion 1999 July 11, in days for each SWAN image.

$r$ : heliocentric distance (AU).

$\Delta$ : geocentric distance (AU).

$g$ : solar Lyman- $\alpha$ g-factor (photons s ${ }^{-1}$ ).

$Q:$ water production rates for each image $\left(\mathrm{s}^{-1}\right)$.

$\Delta Q: 1 \sigma$ formal uncertainty $\left(\mathrm{s}^{-1}\right)$.

post-perihelion. The SWAS observations indicate that the gas production rate has a fairly steep fall-off for distance larger than 1.5 AU. Comet Lee is low in CO (Biver et al. 2000; Mumma et al. 2001b) while its $\mathrm{CH}_{3} \mathrm{OH}$ abundance is enhanced and ethane and acetylene are similar to volatile-rich bright comets such as
Hyakutake and Hale-Bopp. The post-perihelion drop could be indicative of a simple seasonal effect of the nucleus rotation axis exposing different portions of the nucleus surface. It is noteworthy that Biver et al. report $\mathrm{CO}$ depletion pre-perihelion and Mumma et al. report $\mathrm{CO}$ depletion post-perihelion, so it does 


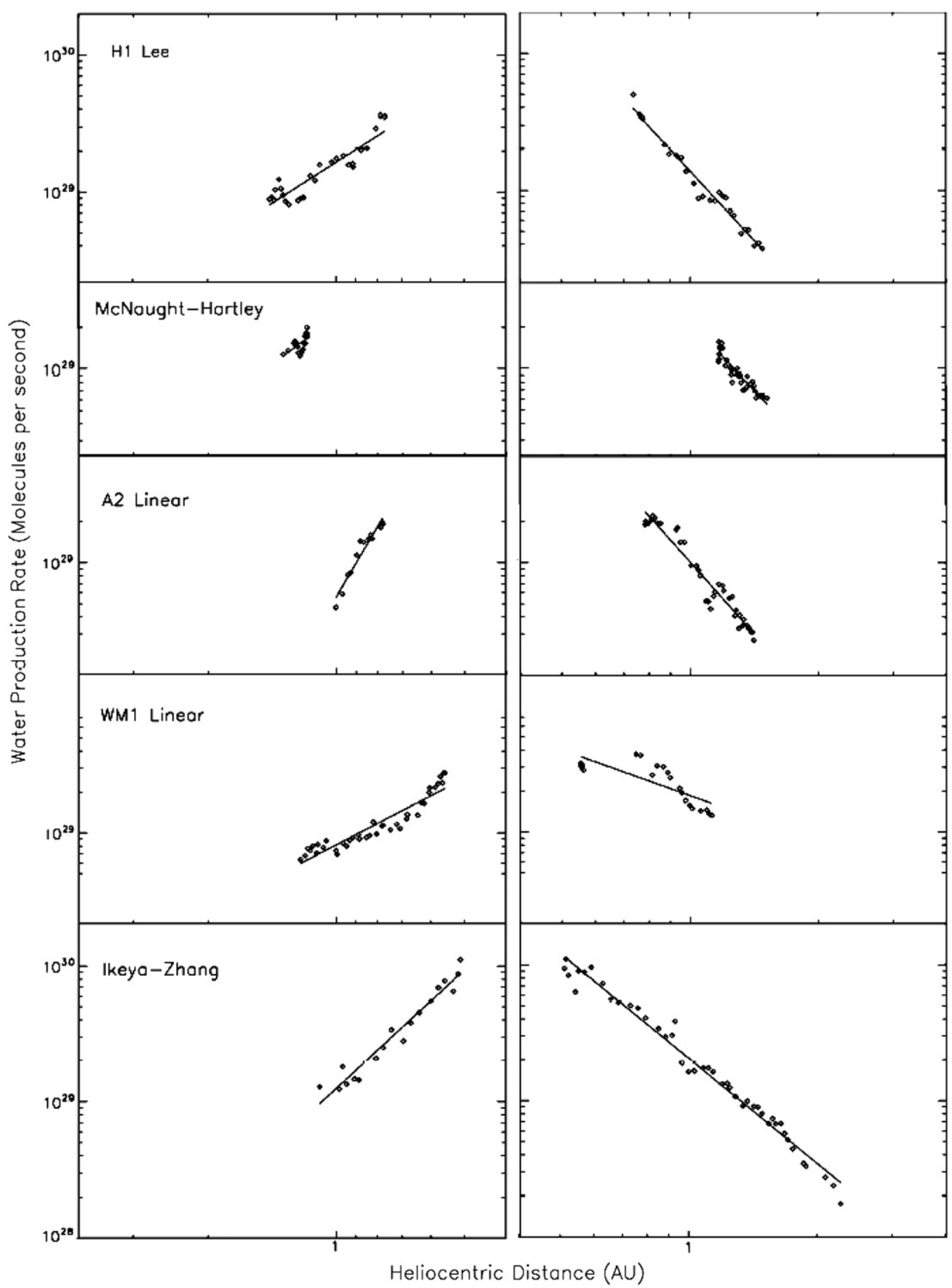

Figure 1. Single-image water production rates and fitted power-law distributions.

not appear that there is a gross change in chemical composition before and after perihelion that would be indicative of gross chemical heterogeneity around the surface.

In the overall SWAN light curve of daily-averaged production rates, there appear to be a few transient features, with small outbursts on the order of $20-40 \%$ increases in gas production rate lasting several days each at -67 days, -46 days, +2 days, and +47 days measured from perihelion. Such variations determined from SWAN observations were corroborated in the case of comet Hyakutake (Bertaux et al. 1998; Combi et al. 2005) which was observed more intensely by many groups than was Lee.

\section{1999 T1 McNAUGHT-HARTLEY}

Comet 1999 T1 McNaught-Hartley (hereafter also referred to as $\mathrm{M}-\mathrm{H}$ ) reached a perihelion distance of $1.1717 \mathrm{AU}$ on 13.4722 December (UT), 2000. SWAN observations of McNaught- 
Table 2

Power-Law Fits to the Water Production Rate Variation

\begin{tabular}{lcccccc}
\hline \hline Comet & $Q_{1}$ & $P$ & $Q_{1}(\mathrm{pre})$ & $P($ pre $)$ & $Q_{1}$ (post) & $P($ post $)$ \\
\hline 1999 H1 Lee & $1.5 \times 10^{29}$ & -2.7 & $1.7 \times 10^{29}$ & $-2.0 \pm 0.2$ & $1.4 \times 10^{29}$ & $-3.4 \pm 0.1$ \\
1999 T1 McNaught-Hartley & $2.9 \times 10^{29}$ & $-3.3^{\mathrm{a}}$ & $2.4 \times 10^{29}$ & $-2.4 \pm 0.7$ & $2.2 \times 10^{29}$ & $-3.3 \pm 0.2$ \\
C 2001 A2 LINEAR & $9.3 \times 10^{28}$ & -4.5 & $5.6 \times 10^{28}$ & $-5.3 \pm 0.4$ & $1.1 \times 10^{29}$ & $-3.7 \pm 0.1$ \\
C 2001 WM1 LINEAR & $1.1 \times 10^{29}$ & -1.4 & $8.2 \times 10^{28}$ & $-1.6 \pm 0.1$ & $1.8 \times 10^{29}$ & $-1.1 \pm 0.2$ \\
P153/Ikeya-Zhang & $1.8 \times 10^{29}$ & -2.7 & $1.2 \times 10^{29}$ & $-2.9 \pm 0.2$ & $2.0 \times 10^{29}$ & $-2.6 \pm 0.1$ \\
C 1995 OI Hale-Bopp & $1.3 \times 10^{31}$ & -2.6 & & & \\
C 1996 B2 Hyakutake & $2.7 \times 10^{29}$ & -2.1 & & &
\end{tabular}

Notes.

a Because of the limited range of heliocentric distance in the data pre-perihelion we have adopted the post-perihelion slope for the "average."

$Q_{1}$ : water production rate at $1 \mathrm{AU}$ : pre and post signify pre-perihelion and post-perihelion.

$P$ : power-law exponent in $r^{p}$ : pre and post signify pre-perihelion and post-perihelion.

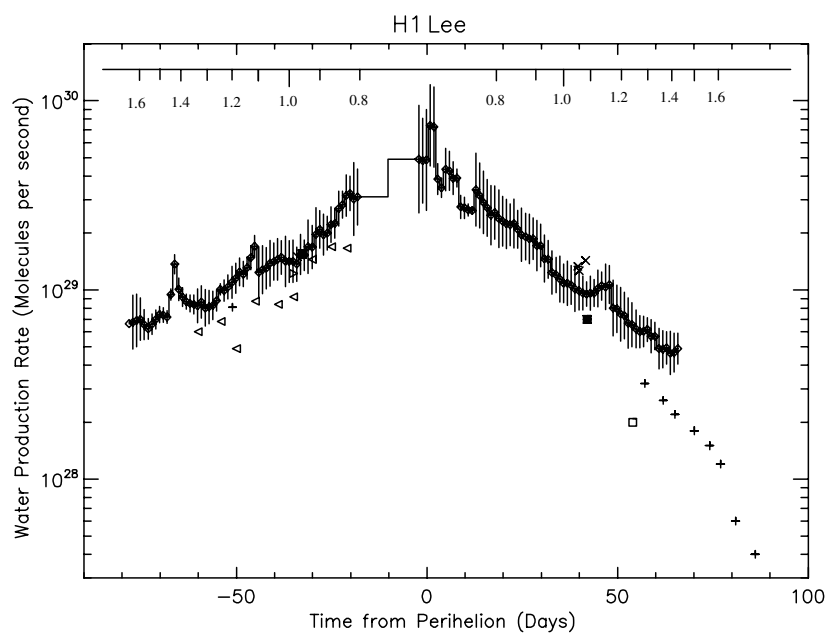

Figure 2. Daily-average water production rates of comet $1999 \mathrm{H} 1$ (Lee). The SWAN results are plotted as small diamonds with vertical lines corresponding to one-sigma uncertainties. The other water production rate values are: left pointing triangles, Biver et al. (2000); right pointing triangle, Lara et al. (2004a); +, Chiu et al. (2001); open square, Weaver et al. (2002); filled square, Feldman et al. (1999); ×, Mumma et al. (2001b) and Dello Russo et al. (2005). The inset scale gives the heliocentric distance in AU.

Hartley are available from early 2000 November to 2001 mid-February. Because of its larger perihelion distance the total length of time when its brightness in Ly- $\alpha$ was high enough to extract good water production rates was limited compared with the other comets in this group. The combination of large heliocentric distance and large production rate made the detectability of $\mathrm{M}-\mathrm{H}$ similar to the other comets in this group at the beginning and end of the run of useful images. The observational circumstances of the individual SWAN images and the single-image water production rates are given in Table 4.

The second panel of Figure 1 shows the results of the singleimage water production rates for McNaught-Hartley plotted as a function of heliocentric distance. Because of the limited heliocentric distance range, especially before perihelion, it is not clear how much significance to place in the power-law exponent before perihelion. The post-perihelion slope is -3.3 . Because of the limited range of heliocentric distances in the pre-perihelion data, we adopted the post-perihelion slope as the "average" value for this comet. The production rate projected back to $1 \mathrm{AU}-$ note that the comet only gets to 1.17 AU-is $\sim 2.3 \times 10^{29} \mathrm{~s}^{-1}$,

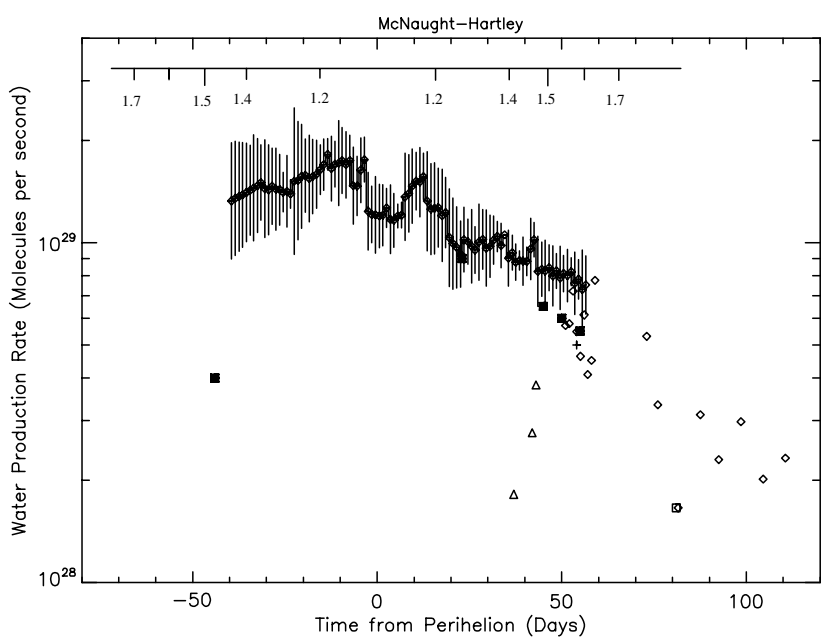

Figure 3. Daily-averaged water production rates of comet 1999 T1 (McNaughtHartley). See Figure 2 caption for details. The other water production rate values are: filled square, Biver et al. (2006); isolated diamonds, Bensch et al. (2004); open square, Weaver et al. (2002); +, Vervack et al. (2004); upward-pointing triangle, Lovell et al. (2008). The inset scale gives the heliocentric distance in AU.

and so while this was not a particularly spectacular object, its gas production rate, if it were at smaller heliocentric distances, is comparable to the more active comets in the group presented here.

Figure 3 and Table 5 give the daily-average water production rates extracted from the whole set of SWAN images as a function of time from perihelion plotted with a number of other measurements yielding water production rates. Again the SWAN values are slightly higher but reasonably consistent with observations by SWAS (Bensch et al. 2004) who observed after perihelion. The post-perihelion radio $\mathrm{OH}$ observations by Biver et al. (2006) are consistent with both SWAS (and SWAN) but the single pre-perihelion measurement 43 days before perihelion is a factor of 3 below the SWAN value. There are other single measurements by Vervack et al. (2004) and Weaver et al. (2002), which are consistent with SWAS and Biver et al. Several values from radio $\mathrm{OH}$ measurements by Lovell et al. (2008) are a factors of 3-5 below the SWAN values, far below the more typical difference of $25 \%$ between SWAN and SWAS or the Biver et al. radio measurements. The other water production rates (mostly from SWAS) extend the SWAN activity light curve out to just past $2 \mathrm{AU}$ after perihelion and indicate that the steep fall-off 
Table 3

Comet Lee: Deconvolved Daily-Averaged Water Production Rates

\begin{tabular}{|c|c|c|}
\hline$\Delta T$ (days) & $Q\left(\mathrm{~s}^{-1}\right)$ & $\Delta Q\left(\mathrm{~s}^{-1}\right)$ \\
\hline-78.173 & $6.633 \times 10^{28}$ & $2.946 \times 10^{28}$ \\
\hline-77.173 & $6.746 \times 10^{28}$ & $2.609 \times 10^{28}$ \\
\hline-76.173 & $6.905 \times 10^{28}$ & $2.607 \times 10^{28}$ \\
\hline-75.173 & $7.002 \times 10^{28}$ & $2.058 \times 10^{28}$ \\
\hline-74.173 & $6.547 \times 10^{28}$ & $1.319 \times 10^{28}$ \\
\hline-73.173 & $6.223 \times 10^{28}$ & $8.458 \times 10^{27}$ \\
\hline-72.173 & $6.588 \times 10^{28}$ & $8.765 \times 10^{27}$ \\
\hline-71.173 & $6.984 \times 10^{28}$ & $8.657 \times 10^{27}$ \\
\hline-70.173 & $7.413 \times 10^{28}$ & $7.613 \times 10^{27}$ \\
\hline-69.173 & $7.357 \times 10^{28}$ & $6.849 \times 10^{27}$ \\
\hline-68.173 & $7.183 \times 10^{28}$ & $5.934 \times 10^{27}$ \\
\hline-67.173 & $9.443 \times 10^{28}$ & $6.688 \times 10^{27}$ \\
\hline-66.173 & $1.368 \times 10^{29}$ & $1.683 \times 10^{28}$ \\
\hline-65.173 & $1.008 \times 10^{29}$ & $1.466 \times 10^{28}$ \\
\hline-64.173 & $9.165 \times 10^{28}$ & $1.101 \times 10^{28}$ \\
\hline-63.173 & $8.675 \times 10^{28}$ & $8.351 \times 10^{27}$ \\
\hline-62.173 & $8.485 \times 10^{28}$ & $9.305 \times 10^{27}$ \\
\hline-61.173 & $8.382 \times 10^{28}$ & $1.045 \times 10^{28}$ \\
\hline-60.173 & $8.254 \times 10^{28}$ & $1.882 \times 10^{28}$ \\
\hline-59.173 & $8.634 \times 10^{28}$ & $1.882 \times 10^{28}$ \\
\hline-58.173 & $8.031 \times 10^{28}$ & $1.990 \times 10^{28}$ \\
\hline-57.173 & $8.118 \times 10^{28}$ & $1.825 \times 10^{28}$ \\
\hline-56.173 & $8.269 \times 10^{28}$ & $1.569 \times 10^{28}$ \\
\hline-55.173 & $8.803 \times 10^{28}$ & $1.209 \times 10^{28}$ \\
\hline-54.173 & $1.007 \times 10^{29}$ & $7.047 \times 10^{27}$ \\
\hline-53.173 & $9.925 \times 10^{28}$ & $1.371 \times 10^{28}$ \\
\hline-52.173 & $1.036 \times 10^{29}$ & $1.626 \times 10^{28}$ \\
\hline-51.173 & $1.097 \times 10^{29}$ & $2.342 \times 10^{28}$ \\
\hline-50.173 & $1.153 \times 10^{29}$ & $2.312 \times 10^{28}$ \\
\hline-49.173 & $1.244 \times 10^{29}$ & $2.282 \times 10^{28}$ \\
\hline-48.173 & $1.212 \times 10^{29}$ & $2.011 \times 10^{28}$ \\
\hline-47.173 & $1.306 \times 10^{29}$ & $1.904 \times 10^{28}$ \\
\hline-46.173 & $1.481 \times 10^{29}$ & $2.310 \times 10^{28}$ \\
\hline-45.173 & $1.702 \times 10^{29}$ & $2.387 \times 10^{28}$ \\
\hline-44.173 & $1.240 \times 10^{29}$ & $4.508 \times 10^{28}$ \\
\hline-43.173 & $1.281 \times 10^{29}$ & $4.358 \times 10^{28}$ \\
\hline-42.173 & $1.300 \times 10^{29}$ & $3.718 \times 10^{28}$ \\
\hline-41.173 & $1.383 \times 10^{29}$ & $4.456 \times 10^{28}$ \\
\hline-40.173 & $1.410 \times 10^{29}$ & $3.566 \times 10^{28}$ \\
\hline-39.173 & $1.448 \times 10^{29}$ & $4.179 \times 10^{28}$ \\
\hline-38.173 & $1.487 \times 10^{29}$ & $3.357 \times 10^{28}$ \\
\hline-37.173 & $1.415 \times 10^{29}$ & $5.051 \times 10^{28}$ \\
\hline-36.173 & $1.414 \times 10^{29}$ & $4.090 \times 10^{28}$ \\
\hline-35.173 & $1.415 \times 10^{29}$ & $4.176 \times 10^{28}$ \\
\hline-34.173 & $1.368 \times 10^{29}$ & $3.144 \times 10^{28}$ \\
\hline-33.173 & $1.538 \times 10^{29}$ & $4.246 \times 10^{28}$ \\
\hline-32.173 & $1.526 \times 10^{29}$ & $3.118 \times 10^{28}$ \\
\hline-31.173 & $1.692 \times 10^{29}$ & $6.506 \times 10^{28}$ \\
\hline-30.173 & $1.686 \times 10^{29}$ & $5.056 \times 10^{28}$ \\
\hline-29.173 & $1.969 \times 10^{29}$ & $7.495 \times 10^{28}$ \\
\hline-28.173 & $2.084 \times 10^{29}$ & $5.333 \times 10^{28}$ \\
\hline-27.173 & $1.960 \times 10^{29}$ & $5.546 \times 10^{28}$ \\
\hline-26.173 & $1.993 \times 10^{29}$ & $4.595 \times 10^{28}$ \\
\hline-25.173 & $2.215 \times 10^{29}$ & $5.357 \times 10^{28}$ \\
\hline-24.173 & $2.249 \times 10^{29}$ & $4.423 \times 10^{28}$ \\
\hline-23.173 & $2.693 \times 10^{29}$ & $6.398 \times 10^{28}$ \\
\hline-22.173 & $2.828 \times 10^{29}$ & $5.058 \times 10^{28}$ \\
\hline-21.173 & $3.147 \times 10^{29}$ & $9.038 \times 10^{28}$ \\
\hline-20.173 & $3.240 \times 10^{29}$ & $7.550 \times 10^{28}$ \\
\hline-19.173 & $3.020 \times 10^{29}$ & $1.680 \times 10^{29}$ \\
\hline-18.173 & $3.102 \times 10^{29}$ & $1.254 \times 10^{29}$ \\
\hline-13.173 & $4.549 \times 10^{29}$ & $1.224 \times 10^{30}$ \\
\hline-12.173 & $4.088 \times 10^{29}$ & $1.031 \times 10^{30}$ \\
\hline-11.173 & $4.020 \times 10^{29}$ & $6.531 \times 10^{29}$ \\
\hline-10.173 & $4.069 \times 10^{29}$ & $6.453 \times 10^{29}$ \\
\hline
\end{tabular}

Table 3

(Continued)

\begin{tabular}{|c|c|c|}
\hline$\Delta T$ (days) & $Q\left(\mathrm{~s}^{-1}\right)$ & $\Delta Q\left(\mathrm{~s}^{-1}\right)$ \\
\hline-9.173 & $3.750 \times 10^{29}$ & $5.633 \times 10^{29}$ \\
\hline-8.173 & $3.780 \times 10^{29}$ & $5.486 \times 10^{29}$ \\
\hline-7.173 & $3.504 \times 10^{29}$ & $4.444 \times 10^{29}$ \\
\hline-6.173 & $3.462 \times 10^{29}$ & $3.761 \times 10^{29}$ \\
\hline-5.173 & $3.711 \times 10^{29}$ & $5.429 \times 10^{29}$ \\
\hline-4.173 & $3.764 \times 10^{29}$ & $5.272 \times 10^{29}$ \\
\hline-3.173 & $4.779 \times 10^{29}$ & $4.831 \times 10^{29}$ \\
\hline-2.173 & $4.913 \times 10^{29}$ & $4.550 \times 10^{29}$ \\
\hline-1.173 & $4.820 \times 10^{29}$ & $3.259 \times 10^{29}$ \\
\hline-0.173 & $4.869 \times 10^{29}$ & $4.117 \times 10^{29}$ \\
\hline 0.827 & $7.397 \times 10^{29}$ & $4.721 \times 10^{29}$ \\
\hline 1.827 & $7.267 \times 10^{29}$ & $4.569 \times 10^{29}$ \\
\hline 2.827 & $3.852 \times 10^{29}$ & $7.974 \times 10^{28}$ \\
\hline 3.827 & $3.462 \times 10^{29}$ & $4.382 \times 10^{28}$ \\
\hline 4.827 & $4.337 \times 10^{29}$ & $1.280 \times 10^{29}$ \\
\hline 5.827 & $4.264 \times 10^{29}$ & $1.148 \times 10^{29}$ \\
\hline 6.827 & $3.896 \times 10^{29}$ & $8.525 \times 10^{28}$ \\
\hline 7.827 & $3.905 \times 10^{29}$ & $4.603 \times 10^{28}$ \\
\hline 8.827 & $2.745 \times 10^{29}$ & $4.152 \times 10^{28}$ \\
\hline 9.827 & $2.717 \times 10^{29}$ & $3.745 \times 10^{28}$ \\
\hline 10.827 & $2.645 \times 10^{29}$ & $1.994 \times 10^{28}$ \\
\hline 11.827 & $2.628 \times 10^{29}$ & $1.044 \times 10^{28}$ \\
\hline 12.827 & $3.384 \times 10^{29}$ & $1.894 \times 10^{29}$ \\
\hline 13.827 & $3.153 \times 10^{29}$ & $1.529 \times 10^{29}$ \\
\hline 14.827 & $2.900 \times 10^{29}$ & $1.345 \times 10^{29}$ \\
\hline 15.827 & $2.719 \times 10^{29}$ & $1.121 \times 10^{29}$ \\
\hline 16.827 & $2.483 \times 10^{29}$ & $1.077 \times 10^{29}$ \\
\hline 17.827 & $2.564 \times 10^{29}$ & $9.893 \times 10^{28}$ \\
\hline 18.827 & $2.378 \times 10^{29}$ & $9.951 \times 10^{28}$ \\
\hline 19.827 & $2.310 \times 10^{29}$ & $8.180 \times 10^{28}$ \\
\hline 20.827 & $2.231 \times 10^{29}$ & $7.804 \times 10^{28}$ \\
\hline 21.827 & $2.210 \times 10^{29}$ & $6.429 \times 10^{28}$ \\
\hline 22.827 & $2.242 \times 10^{29}$ & $7.776 \times 10^{28}$ \\
\hline 23.827 & $2.092 \times 10^{29}$ & $5.466 \times 10^{28}$ \\
\hline 24.827 & $1.955 \times 10^{29}$ & $7.520 \times 10^{28}$ \\
\hline 25.827 & $1.915 \times 10^{29}$ & $6.269 \times 10^{28}$ \\
\hline 26.827 & $1.862 \times 10^{29}$ & $6.573 \times 10^{28}$ \\
\hline 27.827 & $1.865 \times 10^{29}$ & $5.352 \times 10^{28}$ \\
\hline 28.827 & $1.713 \times 10^{29}$ & $6.151 \times 10^{28}$ \\
\hline 29.827 & $1.717 \times 10^{29}$ & $5.033 \times 10^{28}$ \\
\hline 30.827 & $1.463 \times 10^{29}$ & $4.857 \times 10^{28}$ \\
\hline 31.827 & $1.451 \times 10^{29}$ & $3.793 \times 10^{28}$ \\
\hline 32.827 & $1.234 \times 10^{29}$ & $3.711 \times 10^{28}$ \\
\hline 33.827 & $1.198 \times 10^{29}$ & $2.861 \times 10^{28}$ \\
\hline 34.827 & $1.140 \times 10^{29}$ & $3.645 \times 10^{28}$ \\
\hline 35.827 & $1.084 \times 10^{29}$ & $2.888 \times 10^{28}$ \\
\hline 36.827 & $1.086 \times 10^{29}$ & $3.377 \times 10^{28}$ \\
\hline 37.827 & $1.062 \times 10^{29}$ & $2.812 \times 10^{28}$ \\
\hline 38.827 & $1.012 \times 10^{29}$ & $3.161 \times 10^{28}$ \\
\hline 39.827 & $9.887 \times 10^{28}$ & $2.607 \times 10^{28}$ \\
\hline 40.827 & $9.629 \times 10^{28}$ & $2.180 \times 10^{28}$ \\
\hline 41.827 & $9.510 \times 10^{28}$ & $1.529 \times 10^{28}$ \\
\hline 42.827 & $9.606 \times 10^{28}$ & $2.238 \times 10^{28}$ \\
\hline 43.827 & $9.637 \times 10^{28}$ & $1.743 \times 10^{28}$ \\
\hline 44.827 & $1.017 \times 10^{29}$ & $2.543 \times 10^{28}$ \\
\hline 45.827 & $1.049 \times 10^{29}$ & $1.979 \times 10^{28}$ \\
\hline 46.827 & $1.038 \times 10^{29}$ & $3.300 \times 10^{28}$ \\
\hline 47.827 & $1.066 \times 10^{29}$ & $2.765 \times 10^{28}$ \\
\hline 48.827 & $8.011 \times 10^{28}$ & $3.233 \times 10^{28}$ \\
\hline 49.827 & $7.950 \times 10^{28}$ & $2.671 \times 10^{28}$ \\
\hline 50.827 & $7.449 \times 10^{28}$ & $2.880 \times 10^{28}$ \\
\hline 51.827 & $7.318 \times 10^{28}$ & $2.394 \times 10^{28}$ \\
\hline 52.827 & $6.678 \times 10^{28}$ & $2.405 \times 10^{28}$ \\
\hline 53.827 & $6.551 \times 10^{28}$ & $2.005 \times 10^{28}$ \\
\hline 54.827 & $6.216 \times 10^{28}$ & $1.622 \times 10^{28}$ \\
\hline
\end{tabular}


Table 3

(Continued)

\begin{tabular}{lcc}
\hline \hline$\Delta T$ (days) & $Q\left(\mathrm{~s}^{-1}\right)$ & $\Delta Q\left(\mathrm{~s}^{-1}\right)$ \\
\hline 55.827 & $6.015 \times 10^{28}$ & $1.308 \times 10^{28}$ \\
56.827 & $6.037 \times 10^{28}$ & $1.228 \times 10^{28}$ \\
57.827 & $6.167 \times 10^{28}$ & $9.691 \times 10^{27}$ \\
58.827 & $5.682 \times 10^{28}$ & $1.265 \times 10^{28}$ \\
59.827 & $5.699 \times 10^{28}$ & $1.038 \times 10^{28}$ \\
60.827 & $4.893 \times 10^{28}$ & $1.382 \times 10^{28}$ \\
61.827 & $4.861 \times 10^{28}$ & $1.224 \times 10^{28}$ \\
62.827 & $4.930 \times 10^{28}$ & $1.071 \times 10^{28}$ \\
63.827 & $4.639 \times 10^{28}$ & $1.401 \times 10^{28}$ \\
64.827 & $4.665 \times 10^{28}$ & $1.247 \times 10^{28}$ \\
65.827 & $4.896 \times 10^{28}$ & $1.013 \times 10^{28}$ \\
\hline
\end{tabular}

Notes.

$\Delta T$ : time from perihelion 1999 July 11, in days for each deconvolved value.

$Q$ : water production rates for each image $\left(\mathrm{s}^{-1}\right)$. $\Delta Q: 1 \sigma$ formal uncertainty $\left(\mathrm{s}^{-1}\right)$.

of production rate seen in the SWAN data continues to become even steeper at larger heliocentric distances.

Comet McNaught-Hartley was relatively $\mathrm{CO}$ rich. Biver et al. (2006) report a $\mathrm{CO} / \mathrm{H}_{2} \mathrm{O}$ ratio of $15 \%$, which is comparable to the highest values like comet Hale-Bopp. Others of the principal minor species $\left(\mathrm{CH}_{3} \mathrm{OH}, \mathrm{H}_{2} \mathrm{CO}\right.$, and $\left.\mathrm{HCN}\right)$ are fairly nominal and slightly underabundant compared with Hale-Bopp. What seems remarkable about McNaught-Hartley is the combination of high $\mathrm{CO}$ abundance with as steep fall-off of water production with heliocentric distance. The high $\mathrm{CO}$ production rate comets such as 1995 O1 Hale-Bopp and 1996 B2 Hyakutake have moderate slopes, in the range of -2 . Usually, less productive short-period Jupiter family comets have steep slopes and are not usually know to have high $\mathrm{CO}$ abundances.

Finally, the daily-averaged water production rate values show some minor temporal structure, but only at the $\sim 20 \%$ level with increases (outbursts?) 10 days and 41 days after perihelion. While the post-perihelion variation otherwise follows the monotonic long-term power law, the limited pre-perihelion shape seems to be dominated by some irregular structure, but not outbursts. Perhaps this is an indication of a seasonal change in the pole orientation during the pre-perihelion leg around 30 days before perihelion.

\section{2001 A2 LINEAR}

Comet C/2001 A2 (LINEAR) (hereafter also referred to as A2) is noteworthy because of its spectacular splitting events (Jehin et al. 2002; Boehnhardt 2002; Sekanina et al. 2002; Furusho et al. 2003). It reached a perihelion distance of 0.779 AU on 24.5237 May (UT), 2001. SWAN observational results are available from 2001 late-March through the end of 2001 July covering heliocentric distances from $1.36 \mathrm{AU}$ before perihelion to $1.41 \mathrm{AU}$ after perihelion. Table 6 shows the observational circumstances and single-image water production rates using the TRM.

The third panel of Figure 1 shows the single-image water production rates for $\mathrm{A} 2$ plotted as a function of heliocentric distance. The production rates were somewhat lower than the other comets in the group presented here, limiting the length of time coverage of the observations and the penultimate heliocentric distances. Also plotted are the straight lines corresponding to the power-law fits to the production rate variation with heliocentric

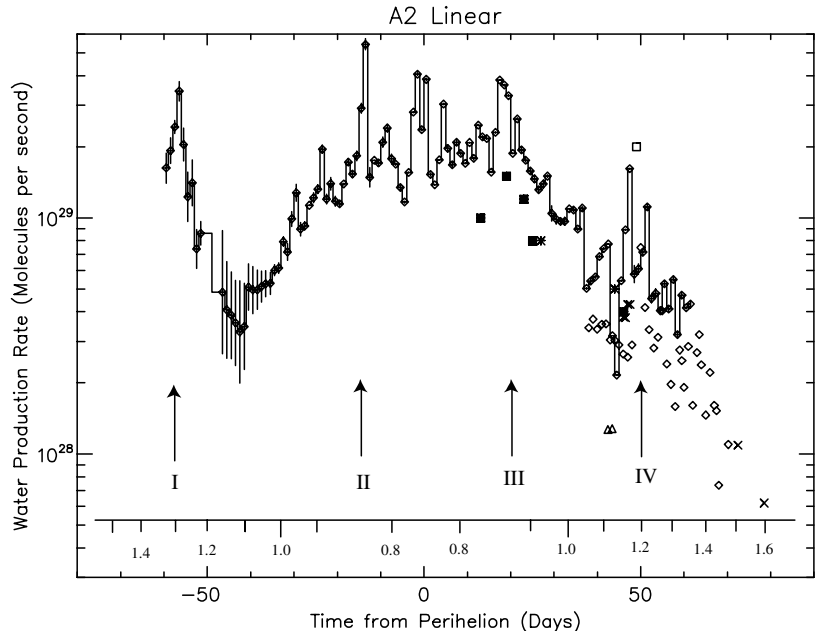

Figure 4. Daily-averaged water production rates of comet $\mathrm{C} / 2001$ A2 (LINEAR). See Figure 2 caption for details. The other water production rate values are: isolated diamonds, Bensch et al. (2004); filled squares, Biver et al. (2006); upward-pointing triangles, Lovell et al. (2008); $\times$, Bonev/Mumma/Dello Russo; open square, Weaver et al. (2002). The inset scale gives the heliocentric distance in AU. The Roman numerals I through IV mark the outburst events noted by Sekanina et al. (2002).

distance. The values are given in the third row of Table 2. As will be discussed below, the production rate variation is highly asymmetric about perihelion. For A2 we have calculated the pre-perihelion power law only for the data after the large outburst around 55 days before perihelion dissipated. Otherwise the variation is nearly flat and does not represent the orderly variation of gas production. Without the early outburst the power law is steeper before perihelion $(-5.3)$ than after $(-3.7)$ and the overall magnitude of gas production after perihelion $(5.6 \times$ $10^{28} \mathrm{~s}^{-1}$ at $\left.1 \mathrm{AU}\right)$ is nearly a factor of 2 smaller than before $\left(1.1 \times 10^{29} \mathrm{~s}^{-1}\right.$ at $\left.1 \mathrm{AU}\right)$. We will return to the discussion of the overall activity level variation below in the context of the outbursts and splitting events.

In Figure 4 and Table 7 we present the daily-averaged production rates for $\mathrm{A} 2$, and they are compared with a number of water production rate results by other investigators. Nearly all of the other observations were during the post-perihelion period. SWAS (Bensch \& Melnick 2006) observed C/2001 A2 (LINEAR) after perihelion beginning on 2001 June 29 when the comet was at a heliocentric distance of $1.035 \mathrm{AU}$ and ending on 2001 August 2 when the comet was at 1.497 AU, extending a week past the last SWAN observation. The SWAS water production rates are about $50 \%$ below the SWAN values before the large outburst in early July after which the overlapping SWAN and SWAS values are consistent to within the scatter. The SWAS measurements then continue on the same trend line for times after the last SWAN observation. These later SWAS values are consistent with the later measurements from infrared water observations (Dello Russo et al. 2005; Bonev et al. 2004), which themselves are consistent with the earlier SWAN measurements. Weaver et al. (2002) reported a water production rate from Far Ultraviolet Spectroscopic Explorer (FUSE) observations of $\mathrm{H}_{2}, \mathrm{H}$, and $\mathrm{OI}$ in $\mathrm{A} 2$ around the time of the large outburst 48 days after perihelion and that single value is a factor of 4 higher than all other values during the same time period. While it is possible that FUSE was seeing an especially enhanced column in its relatively smaller aperture right near the outburst, the TRM de- 
Table 4

Comet McNaught-Hartley: Observational Circumstances and Single-Image Water Production Rates

\begin{tabular}{|c|c|c|c|c|c|}
\hline$\Delta T$ (days) & $r(\mathrm{AU})$ & $\Delta(\mathrm{AU})$ & $g\left(\mathrm{~s}^{-1}\right)$ & $Q\left(\mathrm{~s}^{-1}\right)$ & $\Delta Q\left(\mathrm{~s}^{-1}\right)$ \\
\hline-40.428 & 1.333 & 1.910 & 0.002230 & $1.270 \times 10^{29}$ & $1.732 \times 10^{26}$ \\
\hline-35.475 & 1.298 & 1.884 & 0.002217 & $1.360 \times 10^{29}$ & $1.427 \times 10^{26}$ \\
\hline-29.101 & 1.259 & 1.847 & 0.002191 & $1.527 \times 10^{29}$ & $1.400 \times 10^{26}$ \\
\hline-27.714 & 1.251 & 1.838 & 0.002191 & $1.586 \times 10^{29}$ & $1.315 \times 10^{26}$ \\
\hline-26.651 & 1.245 & 1.832 & 0.002191 & $1.554 \times 10^{29}$ & $1.283 \times 10^{26}$ \\
\hline-24.377 & 1.234 & 1.816 & 0.002181 & $1.445 \times 10^{29}$ & $1.364 \times 10^{26}$ \\
\hline-22.895 & 1.226 & 1.806 & 0.002181 & $1.298 \times 10^{29}$ & $1.448 \times 10^{26}$ \\
\hline-20.534 & 1.216 & 1.790 & 0.002170 & $1.234 \times 10^{29}$ & $1.781 \times 10^{26}$ \\
\hline-18.411 & 1.207 & 1.774 & 0.002161 & $1.314 \times 10^{29}$ & $1.355 \times 10^{26}$ \\
\hline-15.929 & 1.199 & 1.755 & 0.002152 & $1.377 \times 10^{29}$ & $1.336 \times 10^{26}$ \\
\hline-11.905 & 1.187 & 1.723 & 0.002143 & $1.513 \times 10^{29}$ & $1.244 \times 10^{26}$ \\
\hline-10.836 & 1.184 & 1.714 & 0.002136 & $1.736 \times 10^{29}$ & $1.238 \times 10^{26}$ \\
\hline-9.502 & 1.181 & 1.703 & 0.002136 & $1.534 \times 10^{29}$ & $1.408 \times 10^{26}$ \\
\hline-7.114 & 1.177 & 1.682 & 0.002130 & $1.820 \times 10^{29}$ & $2.366 \times 10^{25}$ \\
\hline-5.643 & 1.175 & 1.670 & 0.002130 & $1.776 \times 10^{29}$ & $1.251 \times 10^{26}$ \\
\hline-3.751 & 1.173 & 1.653 & 0.002124 & $1.821 \times 10^{29}$ & $9.894 \times 10^{25}$ \\
\hline-3.132 & 1.173 & 1.647 & 0.002124 & $2.002 \times 10^{29}$ & $2.098 \times 10^{25}$ \\
\hline-0.399 & 1.172 & 1.623 & 0.002121 & $1.708 \times 10^{29}$ & $1.059 \times 10^{26}$ \\
\hline 0.201 & 1.172 & 1.617 & 0.002121 & $1.551 \times 10^{29}$ & $2.581 \times 10^{25}$ \\
\hline 1.650 & 1.172 & 1.604 & 0.002120 & $1.108 \times 10^{29}$ & $1.837 \times 10^{26}$ \\
\hline 5.201 & 1.175 & 1.571 & 0.002117 & $1.157 \times 10^{29}$ & $3.205 \times 10^{25}$ \\
\hline 6.596 & 1.176 & 1.558 & 0.002115 & $1.266 \times 10^{29}$ & $1.393 \times 10^{26}$ \\
\hline 8.665 & 1.180 & 1.539 & 0.002114 & $1.424 \times 10^{29}$ & $1.404 \times 10^{26}$ \\
\hline 9.761 & 1.182 & 1.529 & 0.002114 & $1.391 \times 10^{29}$ & $2.714 \times 10^{25}$ \\
\hline 10.581 & 1.184 & 1.521 & 0.002114 & $1.421 \times 10^{29}$ & $1.046 \times 10^{26}$ \\
\hline 13.610 & 1.191 & 1.493 & 0.002112 & $1.525 \times 10^{29}$ & $1.187 \times 10^{26}$ \\
\hline 15.679 & 1.198 & 1.475 & 0.002113 & $1.398 \times 10^{29}$ & $1.203 \times 10^{26}$ \\
\hline 20.625 & 1.216 & 1.432 & 0.002116 & $1.038 \times 10^{29}$ & $1.490 \times 10^{26}$ \\
\hline 22.368 & 1.224 & 1.417 & 0.002116 & $1.141 \times 10^{29}$ & $1.441 \times 10^{26}$ \\
\hline 27.577 & 1.250 & 1.377 & 0.002124 & $9.930 \times 10^{28}$ & $1.323 \times 10^{26}$ \\
\hline 28.082 & 1.253 & 1.373 & 0.002124 & $9.054 \times 10^{28}$ & $2.707 \times 10^{25}$ \\
\hline 29.522 & 1.261 & 1.363 & 0.002124 & $7.964 \times 10^{28}$ & $1.408 \times 10^{26}$ \\
\hline 31.634 & 1.274 & 1.350 & 0.002132 & $9.308 \times 10^{28}$ & $1.213 \times 10^{26}$ \\
\hline 35.057 & 1.295 & 1.330 & 0.002141 & $9.918 \times 10^{28}$ & $1.154 \times 10^{26}$ \\
\hline 36.112 & 1.302 & 1.324 & 0.002141 & $8.994 \times 10^{28}$ & $1.144 \times 10^{26}$ \\
\hline 37.397 & 1.311 & 1.318 & 0.002141 & $8.829 \times 10^{28}$ & $2.531 \times 10^{25}$ \\
\hline 38.190 & 1.317 & 1.314 & 0.002150 & $8.950 \times 10^{28}$ & $1.202 \times 10^{26}$ \\
\hline 39.257 & 1.324 & 1.310 & 0.002150 & $7.947 \times 10^{28}$ & $1.252 \times 10^{26}$ \\
\hline 40.955 & 1.337 & 1.303 & 0.002150 & $6.990 \times 10^{28}$ & $1.619 \times 10^{26}$ \\
\hline 43.022 & 1.352 & 1.297 & 0.002162 & $7.118 \times 10^{28}$ & $1.502 \times 10^{26}$ \\
\hline 44.277 & 1.362 & 1.293 & 0.002162 & $7.364 \times 10^{28}$ & $2.621 \times 10^{25}$ \\
\hline 45.095 & 1.368 & 1.291 & 0.002162 & $8.859 \times 10^{28}$ & $1.191 \times 10^{26}$ \\
\hline 47.842 & 1.390 & 1.287 & 0.002174 & $7.733 \times 10^{28}$ & $1.242 \times 10^{26}$ \\
\hline 49.915 & 1.407 & 1.285 & 0.002173 & $8.115 \times 10^{28}$ & $1.110 \times 10^{26}$ \\
\hline 51.124 & 1.418 & 1.285 & 0.002173 & $7.546 \times 10^{28}$ & $2.859 \times 10^{25}$ \\
\hline 51.983 & 1.425 & 1.285 & 0.002173 & $6.838 \times 10^{28}$ & $1.210 \times 10^{26}$ \\
\hline 53.038 & 1.434 & 1.286 & 0.002186 & $6.135 \times 10^{28}$ & $1.427 \times 10^{26}$ \\
\hline 54.735 & 1.449 & 1.288 & 0.002186 & $6.372 \times 10^{28}$ & $1.339 \times 10^{26}$ \\
\hline 56.803 & 1.467 & 1.292 & 0.002185 & $6.351 \times 10^{28}$ & $1.333 \times 10^{26}$ \\
\hline 57.854 & 1.477 & 1.294 & 0.002185 & $6.351 \times 10^{28}$ & $1.303 \times 10^{26}$ \\
\hline 59.755 & 1.494 & 1.300 & 0.002199 & $6.252 \times 10^{28}$ & $1.277 \times 10^{26}$ \\
\hline 62.792 & 1.523 & 1.312 & 0.002198 & $6.128 \times 10^{28}$ & $1.336 \times 10^{26}$ \\
\hline
\end{tabular}

Notes.

$\Delta T$ : time from perihelion 2000 December 13, in days for each SWAN image.

$r$ : heliocentric distance (AU).

$\Delta$ : geocentric distance (AU).

$g$ : solar Lyman- $\alpha$-factor (photons $\mathrm{s}^{-1}$ ).

$Q$ : water production rates for each image $\left(\mathrm{s}^{-1}\right)$.

$\Delta Q: 1 \sigma$ formal uncertainty $\left(\mathrm{s}^{-1}\right)$.

convolution of the water production at the nucleus shows some more structure in the variation within a day before the FUSE measurement and another just after the maximum value reg- istered by SWAS. Radio measurements of $\mathrm{OH}$ by Biver et al. (2006) are in good agreement with the SWAN results except for one radio measurement 10 days after perihelion that is a factor 
of 2 below the SWAN results. The radio $\mathrm{OH}$ measurements by Lovell et al. (2008) at 41 days after perihelion are a factor of 3 or more below both SWAN and the other measurements.

Sekanina et al. (2002) have made a careful study of images of released fragments of A2 as well as associated outbursts of activity seen in its visual magnitude record. Our nearly continuous run of daily-averaged water production rates from SWAN provide yet a different comparison, where the TRM deconvolution process potentially places the initial timing of the outburst better than its response as seen in a global visual magnitude estimate. The large outbursts marked number I through IV by Sekanina et al. are all clearly seen in the SWAN results and the outburst IV was also seen in the SWAS results. Both the visual light curve of Sekanina et al. and SWAS measurements delineate an outburst at time 50-51 days after perihelion and is between two peaks recorded in the dailyaveraged deconvolution of the SWAN data, with the second peak somewhat closer to the light curve/SWAS peak. The first of the two peaks is seen at 47.5 days after perihelion. The first peak is very close to the one large value from the HST (Weaver et al. 2002) that probably not coincidentally was taken with a very small aperture, which naturally decreases the time smear and time lag. Extra irregular features were seen in synthetic image deconvolution testing shown in the model (Mäkinen \& Combi 2005) using a Hyakutake-like outburst which was about as large as this one and when field stars and longer and irregular time gaps between images were present in the input dataset.

An interesting comparison with the visual light curve (Sekanina et al. 2002) can also be made with the SWAN results. If one simply converts the water production rate to be proportional to the absolute visual magnitude and then scales it to best match the visual light curve, the entire water production variation from the deconvolved TRM results matches quite well the entire visual light curve except for the period corresponding to the rise of the very first outburst (I) before $T=-55$ days. While the water production rate increases by a factor of 5 from the peak at $T=-65$ days to the first points at -54 days, the visual magnitude increases by orders of magnitude. The interpretation being that the water production outburst and accompanying fragmentation seriously disrupted the nucleus so that the dust production (or more likely the fine dust production) increased precipitously from the time period just before the outburst. After that the dust production, as indicated by the visual magnitude, followed the gas production in a reasonably consistent and proportional manner.

While the power-law exponent for A2 was the steepest of this group, both pre- $(-5.3)$ and post- $(-3.7)$ perihelion, its volatile composition was rather nominal for an Oort cloud comet. Abundances of $\mathrm{CO}$ and $\mathrm{CH}_{3} \mathrm{OH}$ were found to be $1.5 \%$ and $4.0 \%$ respectively, while $\mathrm{CH}_{4}, \mathrm{C}_{2} \mathrm{H}_{6}, \mathrm{C}_{2} \mathrm{H}_{2}$ and $\mathrm{HCN}$ were enhanced (Magee-Sauer et al. 2008; Biver et al. 2006) from typical Oort cloud comets. The continuous set of outbursts accompanied by the release of fragments as well as gas arcs (Boehnhardt 2002) is similar in many ways to Hyakutake (Harris et al. 1997). However, the very steep fall-off of production rate is not a characteristic of Hyakutake whose power-law exponent was close to -2 (Combi et al. 2005). There also is the case of C/1999 S4 (LINEAR), which varied with an exponent close to -2 before perihelion (Mäkinen et al. 2001) with sporadic outbursts and fragment releases only to completely disintegrate shortly after perihelion (Weaver et al. 2001). Comparing these three cases, Hyakutake was a moderately high $\mathrm{CO}$ comet, and $\mathrm{S} 4$ and $\mathrm{A} 2$ are low $\mathrm{CO}$ comets. All three comets seem

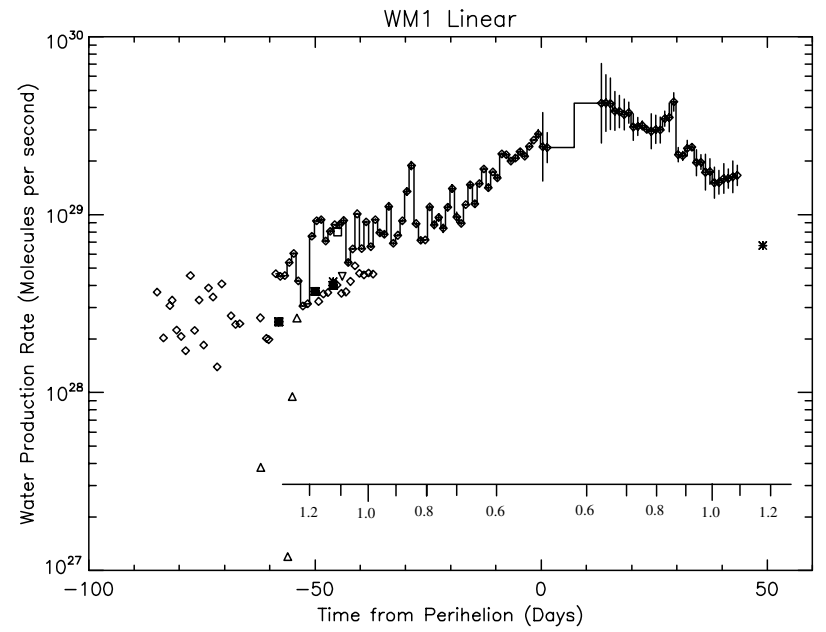

Figure 5. Daily-averaged water production rates of comet C/2001 WM1 (LINEAR). See Figure 2 caption for details. The other water production rate values are: isolated diamonds, Bensch et al. (2004); filled squares, Biver et al. (2006); upward-pointing triangles, Lovell et al. (2008); asterisks, Lecacheux et al. (2003); downward-pointing triangle, Schleicher. The inset scale gives the heliocentric distance in AU.

to have had morphological structures that lent themselves to eruptive fragmentation, while their variations of sublimation with heliocentric distance were quite different from one another and were not at all correlated with their different volatile abundances.

\section{2000 WM1 LINEAR}

Comet 2000 WM1 LINEAR (hereafter also referred to as WM1) reached a perihelion distance of $0.555 \mathrm{AU}$ on 22.6734 January (UT), 2002. SWAN observations of WM1 are available from 2001 December 10 to 2002 March 10, covering a range of heliocentric distances from 1.21 AU before perihelion to 1.12 AU after. The observational circumstances of the individual SWAN images as well as the single-image water production rates are given in Table 8.

The fourth panel of Figure 1 shows the variation of the singleimage water production rates plotted as a function heliocentric distance. While not the most productive comet of this group, WM1 had the shallowest variation with heliocentric distance with power-law exponents before and after perihelion of -1.6 and -1.1 , respectively. The very shallow slope after perihelion appears to be caused by large jump in production during an unfortunate placed data gap between a few images just after perihelion and the beginning of a continuous sequence beginning at about $0.75 \mathrm{AU}$. The rest of the post-perihelion variation beginning at $0.75 \mathrm{AU}$ in fact has a slope similar to the -1.4 of the pre-perihelion variation, but its value at $1 \mathrm{AU}$ is more than a factor of 2 larger than before perihelion. The most likely cause of this is a seasonal variation because of a change in the orientation of the spin axis sometime during the period of January 26 and February 13. An increase such as this could be due to the first exposure of a particularly active region into the dayside hemisphere from an earlier winter polar region or a change in the projected dayside area because of a highly aspherical nucleus, i.e. the Sun exposing a long axis after perihelion but a short axis before perihelion.

Figure 5 shows the daily-average water production rates in C/2001 WM1 from the TRM analysis of the SWAN images plotted as a function of time from perihelion. They are listed 
Table 5

Comet McNaught-Hartley: Daily-Averaged Deconvolved Water Production Rates

\begin{tabular}{|c|c|c|}
\hline$\Delta T$ (days) & $Q\left(\mathrm{~s}^{-1}\right)$ & $\Delta Q\left(\mathrm{~s}^{-1}\right)$ \\
\hline-42.447 & $1.287 \times 10^{29}$ & $5.721 \times 10^{28}$ \\
\hline-41.447 & $1.306 \times 10^{29}$ & $5.299 \times 10^{28}$ \\
\hline-40.447 & $1.330 \times 10^{29}$ & $5.022 \times 10^{28}$ \\
\hline-39.447 & $1.329 \times 10^{29}$ & $6.424 \times 10^{28}$ \\
\hline-38.447 & $1.351 \times 10^{29}$ & $6.364 \times 10^{28}$ \\
\hline-37.447 & $1.368 \times 10^{29}$ & $6.122 \times 10^{28}$ \\
\hline-36.447 & $1.383 \times 10^{29}$ & $5.899 \times 10^{28}$ \\
\hline-35.447 & $1.407 \times 10^{29}$ & $5.574 \times 10^{28}$ \\
\hline-34.447 & $1.429 \times 10^{29}$ & $5.092 \times 10^{28}$ \\
\hline-33.447 & $1.451 \times 10^{29}$ & $6.270 \times 10^{28}$ \\
\hline-32.447 & $1.474 \times 10^{29}$ & $5.530 \times 10^{28}$ \\
\hline-31.447 & $1.504 \times 10^{29}$ & $4.387 \times 10^{28}$ \\
\hline-30.447 & $1.444 \times 10^{29}$ & $5.668 \times 10^{28}$ \\
\hline-29.447 & $1.433 \times 10^{29}$ & $5.119 \times 10^{28}$ \\
\hline-28.447 & $1.467 \times 10^{29}$ & $4.327 \times 10^{28}$ \\
\hline-27.447 & $1.438 \times 10^{29}$ & $4.612 \times 10^{28}$ \\
\hline-26.447 & $1.434 \times 10^{29}$ & $3.892 \times 10^{28}$ \\
\hline-25.447 & $1.410 \times 10^{29}$ & $2.010 \times 10^{28}$ \\
\hline-24.447 & $1.418 \times 10^{29}$ & $3.913 \times 10^{28}$ \\
\hline-23.447 & $1.394 \times 10^{29}$ & $1.425 \times 10^{28}$ \\
\hline-22.447 & $1.520 \times 10^{29}$ & $9.766 \times 10^{28}$ \\
\hline-21.447 & $1.526 \times 10^{29}$ & $7.533 \times 10^{28}$ \\
\hline-20.447 & $1.569 \times 10^{29}$ & $6.590 \times 10^{28}$ \\
\hline-19.447 & $1.578 \times 10^{29}$ & $4.456 \times 10^{28}$ \\
\hline-18.447 & $1.547 \times 10^{29}$ & $5.259 \times 10^{28}$ \\
\hline-17.447 & $1.567 \times 10^{29}$ & $4.489 \times 10^{28}$ \\
\hline-16.447 & $1.591 \times 10^{29}$ & $4.138 \times 10^{28}$ \\
\hline-15.447 & $1.643 \times 10^{29}$ & $3.028 \times 10^{28}$ \\
\hline-14.447 & $1.699 \times 10^{29}$ & $3.244 \times 10^{28}$ \\
\hline-13.447 & $1.820 \times 10^{29}$ & $2.045 \times 10^{28}$ \\
\hline-12.447 & $1.653 \times 10^{29}$ & $4.042 \times 10^{28}$ \\
\hline-11.447 & $1.704 \times 10^{29}$ & $2.856 \times 10^{28}$ \\
\hline-10.447 & $1.716 \times 10^{29}$ & $5.745 \times 10^{28}$ \\
\hline-9.447 & $1.747 \times 10^{29}$ & $4.353 \times 10^{28}$ \\
\hline-8.447 & $1.697 \times 10^{29}$ & $4.276 \times 10^{28}$ \\
\hline-7.447 & $1.749 \times 10^{29}$ & $3.279 \times 10^{28}$ \\
\hline-6.447 & $1.474 \times 10^{29}$ & $4.392 \times 10^{28}$ \\
\hline-5.447 & $1.470 \times 10^{29}$ & $3.273 \times 10^{28}$ \\
\hline-4.447 & $1.636 \times 10^{29}$ & $4.008 \times 10^{28}$ \\
\hline-3.447 & $1.758 \times 10^{29}$ & $2.892 \times 10^{28}$ \\
\hline-2.447 & $1.239 \times 10^{29}$ & $3.733 \times 10^{28}$ \\
\hline-1.447 & $1.213 \times 10^{29}$ & $2.573 \times 10^{28}$ \\
\hline-0.447 & $1.210 \times 10^{29}$ & $3.588 \times 10^{28}$ \\
\hline 0.553 & $1.200 \times 10^{29}$ & $2.894 \times 10^{28}$ \\
\hline 1.553 & $1.207 \times 10^{29}$ & $2.781 \times 10^{28}$ \\
\hline 2.553 & $1.266 \times 10^{29}$ & $1.783 \times 10^{28}$ \\
\hline 3.553 & $1.173 \times 10^{29}$ & $3.058 \times 10^{28}$ \\
\hline 4.553 & $1.165 \times 10^{29}$ & $2.332 \times 10^{28}$ \\
\hline 5.553 & $1.200 \times 10^{29}$ & $1.011 \times 10^{28}$ \\
\hline 6.553 & $1.207 \times 10^{29}$ & $1.092 \times 10^{28}$ \\
\hline 7.553 & $1.365 \times 10^{29}$ & $4.707 \times 10^{28}$ \\
\hline 8.553 & $1.398 \times 10^{29}$ & $4.178 \times 10^{28}$ \\
\hline 9.553 & $1.468 \times 10^{29}$ & $4.202 \times 10^{28}$ \\
\hline 10.553 & $1.519 \times 10^{29}$ & $3.280 \times 10^{28}$ \\
\hline 11.553 & $1.510 \times 10^{29}$ & $3.993 \times 10^{28}$ \\
\hline 12.553 & $1.568 \times 10^{29}$ & $2.661 \times 10^{28}$ \\
\hline 13.553 & $1.326 \times 10^{29}$ & $5.312 \times 10^{28}$ \\
\hline 14.553 & $1.256 \times 10^{29}$ & $4.549 \times 10^{28}$ \\
\hline 15.553 & $1.264 \times 10^{29}$ & $4.585 \times 10^{28}$ \\
\hline 16.553 & $1.271 \times 10^{29}$ & $3.894 \times 10^{28}$ \\
\hline 17.553 & $1.204 \times 10^{29}$ & $4.432 \times 10^{28}$ \\
\hline 18.553 & $1.227 \times 10^{29}$ & $3.301 \times 10^{28}$ \\
\hline 19.553 & $1.036 \times 10^{29}$ & $4.079 \times 10^{28}$ \\
\hline 20.553 & $9.953 \times 10^{28}$ & $3.612 \times 10^{28}$ \\
\hline 21.553 & $9.703 \times 10^{28}$ & $3.073 \times 10^{28}$ \\
\hline
\end{tabular}

Table 5

(Continued)

\begin{tabular}{lcc}
\hline \hline$\Delta T($ days $)$ & $Q\left(\mathrm{~s}^{-1}\right)$ & $\Delta Q\left(\mathrm{~s}^{-1}\right)$ \\
\hline 22.553 & $9.260 \times 10^{28}$ & $2.340 \times 10^{28}$ \\
23.553 & $1.022 \times 10^{29}$ & $2.497 \times 10^{28}$ \\
24.553 & $1.008 \times 10^{29}$ & $1.665 \times 10^{28}$ \\
25.553 & $9.774 \times 10^{28}$ & $2.760 \times 10^{28}$ \\
26.553 & $9.523 \times 10^{28}$ & $1.626 \times 10^{28}$ \\
27.553 & $1.005 \times 10^{29}$ & $2.957 \times 10^{28}$ \\
28.553 & $1.023 \times 10^{29}$ & $2.301 \times 10^{28}$ \\
29.553 & $9.658 \times 10^{28}$ & $2.858 \times 10^{28}$ \\
30.553 & $9.781 \times 10^{28}$ & $2.290 \times 10^{28}$ \\
31.553 & $1.019 \times 10^{29}$ & $1.799 \times 10^{28}$ \\
32.553 & $1.044 \times 10^{29}$ & $1.127 \times 10^{28}$ \\
33.553 & $9.820 \times 10^{28}$ & $1.592 \times 10^{28}$ \\
34.553 & $1.057 \times 10^{29}$ & $3.240 \times 10^{26}$ \\
35.553 & $9.015 \times 10^{28}$ & $1.889 \times 10^{28}$ \\
36.553 & $9.360 \times 10^{28}$ & $1.169 \times 10^{28}$ \\
37.553 & $8.765 \times 10^{28}$ & $1.151 \times 10^{28}$ \\
38.553 & $8.879 \times 10^{28}$ & $6.026 \times 10^{27}$ \\
39.553 & $8.831 \times 10^{28}$ & $1.562 \times 10^{28}$ \\
40.553 & $8.820 \times 10^{28}$ & $8.978 \times 10^{27}$ \\
41.553 & $9.598 \times 10^{28}$ & $2.202 \times 10^{28}$ \\
42.553 & $1.022 \times 10^{29}$ & $1.261 \times 10^{28}$ \\
43.553 & $8.241 \times 10^{28}$ & $2.208 \times 10^{28}$ \\
44.553 & $8.365 \times 10^{28}$ & $1.693 \times 10^{28}$ \\
45.553 & $8.266 \times 10^{28}$ & $1.930 \times 10^{28}$ \\
46.553 & $8.442 \times 10^{28}$ & $1.425 \times 10^{28}$ \\
47.553 & $7.985 \times 10^{28}$ & $1.809 \times 10^{28}$ \\
48.553 & $8.271 \times 10^{28}$ & $1.032 \times 10^{28}$ \\
49.553 & $7.886 \times 10^{28}$ & $1.891 \times 10^{28}$ \\
50.553 & $8.121 \times 10^{28}$ & $1.063 \times 10^{28}$ \\
51.553 & $8.012 \times 10^{28}$ & $1.571 \times 10^{28}$ \\
52.553 & $8.222 \times 10^{28}$ & $8.132 \times 10^{27}$ \\
53.553 & $7.603 \times 10^{28}$ & $1.798 \times 10^{28}$ \\
54.553 & $7.807 \times 10^{28}$ & $1.113 \times 10^{28}$ \\
55.553 & $7.284 \times 10^{28}$ & $2.243 \times 10^{28}$ \\
\hline 56.553 & $7.511 \times 10^{28}$ & $1.637 \times 10^{28}$ \\
\hline & &
\end{tabular}

Notes.

$\Delta T$ : time from perihelion 2000 December 13, in days for each deconvolved value.

$Q$ : water production rates for each image $\left(\mathrm{s}^{-1}\right)$.

$\Delta Q: 1 \sigma$ formal uncertainty $\left(\mathrm{s}^{-1}\right)$.

in Table 9. There is some evidence for a few small outbursts of water production 30 days before perihelion and then again 30 days after perihelion, but otherwise the daily-average values follow the pre- and post-perihelion trends in the single-image values. Also shown in the figure are water production rates from a number of other investigators using different emissions from water and water by-products. The SWAS observations (Bensch et al. 2004) partially overlap the early pre-perihelion coverage by SWAN and precede it by three weeks. The values are consistent but generally at the low side of the scatter in the SWAN values from -60 to -35 days and extend the apparent trend in the SWAN results to the earlier times. One value from Schleicher (a private communication reported by Irvine et al. 2003) from ground-based $\mathrm{OH}$ photometry 46 days before perihelion is consistent with the nearby SWAN values. Radio measurements of $\mathrm{OH}$ by Biver et al. (2006) are also consistent with SWAS (and the lower range of the SWAN results) in the early pre-perihelion period, as are $\mathrm{OH}$ observations from the $H S T$ reported by Weaver et al. (2002) and water from ODIN (Lecacheux et al. 2003). One of the results of $\mathrm{OH}$ radio measurements by Lovell et al. (2008) 54 days before perihelion is consistent with the rest, but three other earlier values fall factors of several below. 
Table 6

Comet (LINEAR) A2: Observational Circumstances and Single-Image Water Production Rates

\begin{tabular}{|c|c|c|c|c|c|}
\hline$\Delta T$ (days) & $r(\mathrm{AU})$ & $\Delta(\mathrm{AU})$ & $g\left(\mathrm{~s}^{-1}\right)$ & $Q\left(\mathrm{~s}^{-1}\right)$ & $\Delta Q\left(\mathrm{~s}^{-1}\right)$ \\
\hline-61.000 & 1.360 & 0.946 & 0.002439 & $1.281 \times 10^{29}$ & $6.364 \times 10^{25}$ \\
\hline-58.343 & 1.324 & 0.942 & 0.002438 & $1.967 \times 10^{29}$ & $5.077 \times 10^{25}$ \\
\hline-55.614 & 1.287 & 0.938 & 0.002437 & $2.085 \times 10^{29}$ & $5.041 \times 10^{25}$ \\
\hline-54.559 & 1.273 & 0.936 & 0.002437 & $2.120 \times 10^{29}$ & $5.099 \times 10^{25}$ \\
\hline-53.175 & 1.254 & 0.933 & 0.002436 & $1.688 \times 10^{29}$ & $5.381 \times 10^{25}$ \\
\hline-51.495 & 1.232 & 0.930 & 0.002436 & $1.426 \times 10^{29}$ & $7.498 \times 10^{25}$ \\
\hline-49.448 & 1.205 & 0.925 & 0.002409 & $8.962 \times 10^{28}$ & $1.106 \times 10^{26}$ \\
\hline-47.537 & 1.180 & 0.919 & 0.002409 & $5.900 \times 10^{28}$ & $1.733 \times 10^{26}$ \\
\hline-33.256 & 1.003 & 0.859 & 0.002333 & $4.699 \times 10^{28}$ & $1.470 \times 10^{26}$ \\
\hline-30.235 & 0.969 & 0.841 & 0.002311 & $5.882 \times 10^{28}$ & $1.071 \times 10^{26}$ \\
\hline-27.456 & 0.940 & 0.822 & 0.002289 & $8.120 \times 10^{28}$ & $9.265 \times 10^{25}$ \\
\hline-26.067 & 0.925 & 0.812 & 0.002289 & $8.420 \times 10^{28}$ & $8.933 \times 10^{25}$ \\
\hline-23.054 & 0.896 & 0.789 & 0.002268 & $1.136 \times 10^{29}$ & $6.479 \times 10^{25}$ \\
\hline-21.006 & 0.878 & 0.772 & 0.002248 & $1.438 \times 10^{29}$ & $6.279 \times 10^{25}$ \\
\hline-19.097 & 0.862 & 0.756 & 0.002229 & $1.418 \times 10^{29}$ & $5.314 \times 10^{25}$ \\
\hline-16.084 & 0.839 & 0.728 & 0.002195 & $1.493 \times 10^{29}$ & $5.592 \times 10^{25}$ \\
\hline-14.907 & 0.830 & 0.717 & 0.002181 & $1.602 \times 10^{29}$ & $1.110 \times 10^{25}$ \\
\hline-14.035 & 0.825 & 0.708 & 0.002168 & $1.505 \times 10^{29}$ & $5.580 \times 10^{25}$ \\
\hline-5.397 & 0.786 & 0.616 & 0.002106 & $1.813 \times 10^{29}$ & $4.293 \times 10^{25}$ \\
\hline-1.744 & 0.779 & 0.575 & 0.002087 & $1.910 \times 10^{29}$ & $3.819 \times 10^{25}$ \\
\hline-0.440 & 0.779 & 0.559 & 0.002083 & $1.975 \times 10^{29}$ & $8.121 \times 10^{24}$ \\
\hline 0.298 & 0.779 & 0.551 & 0.002083 & $2.304 \times 10^{29}$ & $2.766 \times 10^{25}$ \\
\hline 2.341 & 0.780 & 0.527 & 0.002080 & $2.421 \times 10^{29}$ & $2.475 \times 10^{25}$ \\
\hline 5.568 & 0.786 & 0.489 & 0.002074 & $1.910 \times 10^{29}$ & $3.036 \times 10^{25}$ \\
\hline 6.386 & 0.788 & 0.479 & 0.002076 & $2.012 \times 10^{29}$ & $7.954 \times 10^{24}$ \\
\hline 7.637 & 0.793 & 0.465 & 0.002076 & $1.935 \times 10^{29}$ & $3.000 \times 10^{25}$ \\
\hline 9.707 & 0.801 & 0.441 & 0.002077 & $1.963 \times 10^{29}$ & $2.798 \times 10^{25}$ \\
\hline 12.395 & 0.815 & 0.410 & 0.002086 & $2.081 \times 10^{29}$ & $2.473 \times 10^{25}$ \\
\hline 13.141 & 0.819 & 0.402 & 0.002093 & $2.210 \times 10^{29}$ & $5.635 \times 10^{24}$ \\
\hline 14.465 & 0.828 & 0.388 & 0.002102 & $2.133 \times 10^{29}$ & $2.238 \times 10^{25}$ \\
\hline 16.396 & 0.841 & 0.367 & 0.002111 & $1.943 \times 10^{29}$ & $2.577 \times 10^{25}$ \\
\hline 18.147 & 0.854 & 0.349 & 0.002122 & $1.947 \times 10^{29}$ & $5.473 \times 10^{24}$ \\
\hline 26.503 & 0.930 & 0.277 & 0.002172 & $1.744 \times 10^{29}$ & $2.215 \times 10^{25}$ \\
\hline 27.126 & 0.936 & 0.273 & 0.002172 & $1.811 \times 10^{29}$ & $4.523 \times 10^{24}$ \\
\hline 28.573 & 0.951 & 0.264 & 0.002186 & $1.417 \times 10^{29}$ & $2.456 \times 10^{25}$ \\
\hline 30.515 & 0.972 & 0.254 & 0.002201 & $1.418 \times 10^{29}$ & $2.272 \times 10^{25}$ \\
\hline 33.560 & 1.007 & 0.244 & 0.002200 & $9.531 \times 10^{28}$ & $2.889 \times 10^{25}$ \\
\hline 36.099 & 1.036 & 0.241 & 0.002215 & $9.466 \times 10^{28}$ & $2.945 \times 10^{25}$ \\
\hline 37.117 & 1.049 & 0.241 & 0.002214 & $8.754 \times 10^{28}$ & $6.652 \times 10^{24}$ \\
\hline 38.031 & 1.060 & 0.242 & 0.002232 & $8.029 \times 10^{28}$ & $3.187 \times 10^{25}$ \\
\hline 40.742 & 1.093 & 0.248 & 0.002231 & $5.206 \times 10^{28}$ & $3.660 \times 10^{25}$ \\
\hline 41.865 & 1.107 & 0.253 & 0.002230 & $5.168 \times 10^{28}$ & $3.522 \times 10^{25}$ \\
\hline 42.921 & 1.120 & 0.258 & 0.002230 & $4.564 \times 10^{28}$ & $3.809 \times 10^{25}$ \\
\hline 44.198 & 1.137 & 0.265 & 0.002248 & $5.676 \times 10^{28}$ & $7.923 \times 10^{24}$ \\
\hline 45.005 & 1.147 & 0.270 & 0.002247 & $6.101 \times 10^{28}$ & $3.918 \times 10^{25}$ \\
\hline 46.765 & 1.170 & 0.282 & 0.002246 & $6.933 \times 10^{28}$ & $1.414 \times 10^{24}$ \\
\hline 48.612 & 1.194 & 0.297 & 0.002245 & $6.801 \times 10^{28}$ & $6.197 \times 10^{24}$ \\
\hline 49.187 & 1.202 & 0.302 & 0.002245 & $6.216 \times 10^{28}$ & $8.003 \times 10^{24}$ \\
\hline 51.922 & 1.238 & 0.328 & 0.002263 & $5.463 \times 10^{28}$ & $4.038 \times 10^{25}$ \\
\hline 53.460 & 1.258 & 0.344 & 0.002262 & $5.617 \times 10^{28}$ & $8.362 \times 10^{24}$ \\
\hline 54.944 & 1.278 & 0.360 & 0.002261 & $4.083 \times 10^{28}$ & $5.015 \times 10^{25}$ \\
\hline 55.481 & 1.285 & 0.366 & 0.002261 & $4.483 \times 10^{28}$ & $2.086 \times 10^{25}$ \\
\hline 56.922 & 1.305 & 0.383 & 0.002260 & $3.289 \times 10^{28}$ & $5.780 \times 10^{25}$ \\
\hline 57.453 & 1.312 & 0.389 & 0.002260 & $4.120 \times 10^{28}$ & $2.301 \times 10^{25}$ \\
\hline 58.918 & 1.332 & 0.407 & 0.002259 & $3.445 \times 10^{28}$ & $5.918 \times 10^{25}$ \\
\hline 59.425 & 1.339 & 0.413 & 0.002259 & $3.830 \times 10^{28}$ & $2.644 \times 10^{25}$ \\
\hline 61.370 & 1.365 & 0.438 & 0.002258 & $3.462 \times 10^{28}$ & $2.306 \times 10^{25}$ \\
\hline 62.252 & 1.377 & 0.449 & 0.002258 & $3.302 \times 10^{28}$ & $8.194 \times 10^{25}$ \\
\hline 63.342 & 1.392 & 0.464 & 0.002257 & $3.115 \times 10^{28}$ & $3.510 \times 10^{25}$ \\
\hline 64.227 & 1.404 & 0.476 & 0.002257 & $3.097 \times 10^{28}$ & $9.048 \times 10^{25}$ \\
\hline 64.906 & 1.414 & 0.485 & 0.002256 & $2.698 \times 10^{28}$ & $5.570 \times 10^{24}$ \\
\hline
\end{tabular}

Notes.

$\Delta T$ : time from perihelion 2001 May 24, in days for each SWAN image; $r$ : heliocentric distance (AU); $\Delta$ : geocentric distance (AU); $g$ : solar Lyman- $\alpha$-factor (photons $\mathrm{s}^{-1}$ ); $Q$ : water production rates for each image $\left(\mathrm{s}^{-1}\right) ; \Delta Q: 1 \sigma$ formal uncertainty $\left(\mathrm{s}^{-1}\right)$. 
Table 7

Comet (LINEAR) A2: Daily-Averaged Deconvolved Water Production Rates

\begin{tabular}{|c|c|c|}
\hline$\Delta T$ (days) & $Q\left(\mathrm{~s}^{-1}\right)$ & $\Delta Q\left(\mathrm{~s}^{-1}\right)$ \\
\hline-63.524 & $1.487 \times 10^{29}$ & $5.853 \times 10^{27}$ \\
\hline-62.524 & $1.586 \times 10^{29}$ & $3.176 \times 10^{28}$ \\
\hline-61.524 & $1.707 \times 10^{29}$ & $3.709 \times 10^{28}$ \\
\hline-60.524 & $1.954 \times 10^{29}$ & $3.249 \times 10^{28}$ \\
\hline-59.524 & $1.626 \times 10^{29}$ & $2.580 \times 10^{28}$ \\
\hline-58.524 & $1.926 \times 10^{29}$ & $2.545 \times 10^{28}$ \\
\hline-57.524 & $2.425 \times 10^{29}$ & $1.537 \times 10^{28}$ \\
\hline-56.524 & $3.441 \times 10^{29}$ & $3.437 \times 10^{28}$ \\
\hline-55.524 & $2.045 \times 10^{29}$ & $3.547 \times 10^{28}$ \\
\hline-54.524 & $1.228 \times 10^{29}$ & $3.340 \times 10^{28}$ \\
\hline-53.524 & $1.406 \times 10^{29}$ & $3.513 \times 10^{28}$ \\
\hline-52.524 & $7.392 \times 10^{28}$ & $1.554 \times 10^{28}$ \\
\hline-51.524 & $8.593 \times 10^{28}$ & $1.053 \times 10^{28}$ \\
\hline-46.524 & $4.846 \times 10^{28}$ & $3.999 \times 10^{28}$ \\
\hline-45.524 & $4.074 \times 10^{28}$ & $2.462 \times 10^{28}$ \\
\hline-44.524 & $3.868 \times 10^{28}$ & $2.040 \times 10^{28}$ \\
\hline-43.524 & $3.593 \times 10^{28}$ & $1.853 \times 10^{28}$ \\
\hline-42.524 & $3.284 \times 10^{28}$ & $2.128 \times 10^{28}$ \\
\hline-41.524 & $3.465 \times 10^{28}$ & $1.806 \times 10^{28}$ \\
\hline-40.524 & $5.093 \times 10^{28}$ & $1.292 \times 10^{28}$ \\
\hline-39.524 & $4.947 \times 10^{28}$ & $1.276 \times 10^{28}$ \\
\hline-38.524 & $4.957 \times 10^{28}$ & $1.050 \times 10^{28}$ \\
\hline-37.524 & $5.117 \times 10^{28}$ & $8.287 \times 10^{27}$ \\
\hline-36.524 & $5.260 \times 10^{28}$ & $7.112 \times 10^{27}$ \\
\hline-35.524 & $5.280 \times 10^{28}$ & $6.035 \times 10^{27}$ \\
\hline-34.524 & $6.002 \times 10^{28}$ & $3.290 \times 10^{27}$ \\
\hline-33.524 & $6.127 \times 10^{28}$ & $3.170 \times 10^{27}$ \\
\hline-32.524 & $7.940 \times 10^{28}$ & $3.761 \times 10^{27}$ \\
\hline-31.524 & $7.170 \times 10^{28}$ & $6.176 \times 10^{27}$ \\
\hline-30.524 & $9.895 \times 10^{28}$ & $7.568 \times 10^{27}$ \\
\hline-29.524 & $1.275 \times 10^{29}$ & $1.091 \times 10^{28}$ \\
\hline-28.524 & $8.947 \times 10^{28}$ & $6.118 \times 10^{27}$ \\
\hline-27.524 & $9.236 \times 10^{28}$ & $3.689 \times 10^{27}$ \\
\hline-26.524 & $1.131 \times 10^{29}$ & $3.576 \times 10^{26}$ \\
\hline-25.524 & $1.218 \times 10^{29}$ & $6.067 \times 10^{27}$ \\
\hline-24.524 & $1.323 \times 10^{29}$ & $5.640 \times 10^{27}$ \\
\hline-23.524 & $1.955 \times 10^{29}$ & $8.223 \times 10^{27}$ \\
\hline-22.524 & $1.200 \times 10^{29}$ & $5.668 \times 10^{27}$ \\
\hline-21.524 & $1.395 \times 10^{29}$ & $8.689 \times 10^{27}$ \\
\hline-20.524 & $1.178 \times 10^{29}$ & $5.850 \times 10^{27}$ \\
\hline-19.524 & $1.147 \times 10^{29}$ & $2.315 \times 10^{27}$ \\
\hline-18.524 & $1.391 \times 10^{29}$ & $1.365 \times 10^{27}$ \\
\hline-17.524 & $1.722 \times 10^{29}$ & $4.988 \times 10^{27}$ \\
\hline-16.524 & $1.531 \times 10^{29}$ & $5.096 \times 10^{27}$ \\
\hline-15.624 & $1.833 \times 10^{29}$ & $8.721 \times 10^{27}$ \\
\hline-14.524 & $2.914 \times 10^{29}$ & $1.458 \times 10^{28}$ \\
\hline-13.524 & $5.432 \times 10^{29}$ & $3.005 \times 10^{28}$ \\
\hline-12.524 & $1.483 \times 10^{29}$ & $1.471 \times 10^{28}$ \\
\hline-11.524 & $1.751 \times 10^{29}$ & $4.827 \times 10^{28}$ \\
\hline-10.524 & $1.706 \times 10^{29}$ & $5.268 \times 10^{28}$ \\
\hline-9.524 & $2.092 \times 10^{29}$ & $1.078 \times 10^{28}$ \\
\hline-8.524 & $2.402 \times 10^{29}$ & $8.202 \times 10^{27}$ \\
\hline-7.524 & $1.778 \times 10^{29}$ & $8.335 \times 10^{27}$ \\
\hline-6.524 & $1.687 \times 10^{29}$ & $1.938 \times 10^{27}$ \\
\hline-5.524 & $1.345 \times 10^{29}$ & $2.937 \times 10^{27}$ \\
\hline-4.524 & $1.167 \times 10^{29}$ & $1.067 \times 10^{27}$ \\
\hline-3.524 & $1.556 \times 10^{29}$ & $7.421 \times 10^{26}$ \\
\hline-2.524 & $2.802 \times 10^{29}$ & $1.731 \times 10^{27}$ \\
\hline-1.524 & $4.060 \times 10^{29}$ & $5.274 \times 10^{28}$ \\
\hline-0.524 & $2.356 \times 10^{29}$ & $3.127 \times 10^{27}$ \\
\hline 0.476 & $3.858 \times 10^{29}$ & $6.092 \times 10^{27}$ \\
\hline 1.476 & $1.528 \times 10^{29}$ & $5.292 \times 10^{27}$ \\
\hline 2.476 & $1.379 \times 10^{29}$ & $2.482 \times 10^{26}$ \\
\hline 3.476 & $1.760 \times 10^{29}$ & $5.404 \times 10^{26}$ \\
\hline 4.476 & $3.035 \times 10^{29}$ & $3.710 \times 10^{27}$ \\
\hline 5.476 & $1.970 \times 10^{29}$ & $2.121 \times 10^{27}$ \\
\hline
\end{tabular}

Table 7

(Continued)

\begin{tabular}{|c|c|c|}
\hline$\Delta T$ (days) & $Q\left(\mathrm{~s}^{-1}\right)$ & $\Delta Q\left(\mathrm{~s}^{-1}\right)$ \\
\hline 6.476 & $1.677 \times 10^{29}$ & $3.932 \times 10^{27}$ \\
\hline 7.476 & $2.087 \times 10^{29}$ & $3.910 \times 10^{27}$ \\
\hline 8.476 & $1.875 \times 10^{29}$ & $2.730 \times 10^{27}$ \\
\hline 9.476 & $1.704 \times 10^{29}$ & $8.398 \times 10^{26}$ \\
\hline 10.476 & $2.085 \times 10^{29}$ & $5.903 \times 10^{26}$ \\
\hline 11.476 & $1.786 \times 10^{29}$ & $2.334 \times 10^{27}$ \\
\hline 12.476 & $2.478 \times 10^{29}$ & $1.921 \times 10^{27}$ \\
\hline 13.476 & $2.209 \times 10^{29}$ & $1.866 \times 10^{27}$ \\
\hline 14.476 & $2.172 \times 10^{29}$ & $1.298 \times 10^{27}$ \\
\hline 20.476 & $1.938 \times 10^{29}$ & $1.900 \times 10^{28}$ \\
\hline 21.476 & $1.839 \times 10^{29}$ & $2.016 \times 10^{28}$ \\
\hline 22.476 & $1.935 \times 10^{29}$ & $6.033 \times 10^{27}$ \\
\hline 23.476 & $1.753 \times 10^{29}$ & $2.676 \times 10^{27}$ \\
\hline 24.476 & $1.580 \times 10^{29}$ & $2.659 \times 10^{27}$ \\
\hline 25.476 & $1.463 \times 10^{29}$ & $4.810 \times 10^{27}$ \\
\hline 26.476 & $1.314 \times 10^{29}$ & $2.994 \times 10^{27}$ \\
\hline 27.476 & $1.389 \times 10^{29}$ & $5.572 \times 10^{27}$ \\
\hline 28.476 & $1.502 \times 10^{29}$ & $1.993 \times 10^{27}$ \\
\hline 29.476 & $1.044 \times 10^{29}$ & $8.110 \times 10^{27}$ \\
\hline 30.476 & $9.871 \times 10^{28}$ & $4.944 \times 10^{27}$ \\
\hline 31.476 & $9.649 \times 10^{28}$ & $2.807 \times 10^{27}$ \\
\hline 32.476 & $9.661 \times 10^{28}$ & $3.807 \times 10^{27}$ \\
\hline 33.476 & $1.093 \times 10^{29}$ & $1.297 \times 10^{26}$ \\
\hline 34.476 & $1.081 \times 10^{29}$ & $1.813 \times 10^{27}$ \\
\hline 35.476 & $8.955 \times 10^{28}$ & $1.235 \times 10^{27}$ \\
\hline 36.476 & $1.099 \times 10^{29}$ & $1.944 \times 10^{27}$ \\
\hline 37.476 & $5.027 \times 10^{28}$ & $3.104 \times 10^{26}$ \\
\hline 38.476 & $5.397 \times 10^{28}$ & $1.356 \times 10^{27}$ \\
\hline 39.476 & $5.616 \times 10^{28}$ & $7.596 \times 10^{26}$ \\
\hline 40.476 & $6.858 \times 10^{28}$ & $9.331 \times 10^{26}$ \\
\hline 41.476 & $7.400 \times 10^{28}$ & $6.203 \times 10^{26}$ \\
\hline 42.476 & $7.761 \times 10^{28}$ & $8.699 \times 10^{25}$ \\
\hline 43.476 & $3.166 \times 10^{28}$ & $2.327 \times 10^{25}$ \\
\hline 44.476 & $2.161 \times 10^{28}$ & $1.010 \times 10^{26}$ \\
\hline 45.476 & $5.426 \times 10^{28}$ & $2.675 \times 10^{26}$ \\
\hline 46.476 & $8.909 \times 10^{28}$ & $4.097 \times 10^{26}$ \\
\hline 47.476 & $1.613 \times 10^{29}$ & $1.019 \times 10^{27}$ \\
\hline 48.476 & $5.777 \times 10^{28}$ & $5.081 \times 10^{27}$ \\
\hline 49.476 & $6.074 \times 10^{28}$ & $3.196 \times 10^{27}$ \\
\hline 50.476 & $7.154 \times 10^{28}$ & $1.812 \times 10^{27}$ \\
\hline 51.476 & $1.113 \times 10^{29}$ & $2.600 \times 10^{27}$ \\
\hline 52.476 & $4.543 \times 10^{28}$ & $1.865 \times 10^{27}$ \\
\hline 53.476 & $4.785 \times 10^{28}$ & $2.204 \times 10^{27}$ \\
\hline 54.476 & $4.043 \times 10^{28}$ & $9.393 \times 10^{26}$ \\
\hline 55.476 & $5.265 \times 10^{28}$ & $8.745 \times 10^{26}$ \\
\hline 56.476 & $4.115 \times 10^{28}$ & $9.656 \times 10^{26}$ \\
\hline 57.476 & $5.485 \times 10^{28}$ & $8.877 \times 10^{26}$ \\
\hline 58.476 & $3.199 \times 10^{28}$ & $8.215 \times 10^{26}$ \\
\hline 59.476 & $4.699 \times 10^{28}$ & $9.039 \times 10^{26}$ \\
\hline 60.476 & $4.159 \times 10^{28}$ & $4.834 \times 10^{26}$ \\
\hline 61.476 & $4.310 \times 10^{28}$ & $1.133 \times 10^{27}$ \\
\hline
\end{tabular}

Notes.

$\Delta T$ : time from perihelion 2001 May 24, in days for each deconvolved value; $Q$ : water production rates for each image $\left(\mathrm{s}^{-1}\right) ; \Delta Q: 1 \sigma$ formal uncertainty $\left(\mathrm{s}^{-1}\right)$.

Biver et al. (2006) and Weaver et al. (2002) report C/2000 WM1 as being $\mathrm{CO}$ poor. In addition it is low in $\mathrm{CH}_{3} \mathrm{OH}$, low but similar to A2 and I-Z, McNaught-Hartley in $\mathrm{H}_{2} \mathrm{CO}$, low in $\mathrm{H}_{2} \mathrm{~S}$ and $\mathrm{CS}$, low and similar to McNaught-Hartley in $\mathrm{HCN}$ (Biver et al. 2006). Lara et al. (2004b) suggest that it is a $\mathrm{C}_{2}$-enriched comet, based on visible range long-slit spectroscopy. They also report noticeable jet activity in both gaseous species and dust, a trait common with Hyakutake, Hale-Bopp, 
and other comets. Therefore, it is quite interesting that in WM1 we have an Oort cloud comet, or perhaps nearly an Oort cloud comet that has passed through the inner solar system before (Lara et al. 2004b), that is definitely depleted in some of the typical volatile components except perhaps the $\mathrm{C}_{2}$ parent, compared with the bright comets Hyakutake and Hale-Bopp but yet displays the most shallow power law in its sublimation rate as a function of heliocentric distance among not only the comets in the group given here but also Hyakutake and Hale-Bopp which are close to the canonical $r^{-2}$ variation. Normally, one would associate a steeper power law with less volatility and a shallower power law with more volatility.

There is some variability in the water production rate over time. This can be seen both in the single-image data as well as the deconvolved daily imaged values. There is evidence for an outburst at 29 days before perihelion that is a factor of 2.5 above the ambient level, as well as 30 days after perihelion that is about $60 \%$ above the ambient level. There were no reported fragments from WM1 as there were for Hyakutake, S4, or C/2001 A2 LINEAR.

\section{153P/IKEYA ZHANG}

Comet 153P/Ikeya-Zhang (hereafter also referred to as I-Z) was initially discovered as a "new" long-period comet and later classified as a periodic comet P153 once its orbit was better determined to have a period of 366.5 years. Typically, it is still classified as an Oort cloud comet (Mumma et al. 2003; Kawakita et al. 2003). Ikeya-Zhang reached a perihelion distance of 0.507 AU on 18.9808 March (UT), 2002. SWAN observations are available from 2002 early-February to 2002 mid-July covering a range of heliocentric distances from 1.09 AU before perihelion to $2.25 \mathrm{AU}$ after perihelion. Table 10 gives the observational circumstances of the individual SWAN images and the single-image water production rates.

The bottom panel of Figure 1 shows the variation of the singleimage water production rates plotted as a function of heliocentric distance. Because of its small perihelion distance and substantial production rate, the SWAN observations cover the largest range of relative heliocentric distance as well as the largest heliocentric distance post-perihelion for any of the comets given here. The variation with heliocentric distance was reasonably symmetric about perihelion with values at $1 \mathrm{AU}$ before and after of 1.2 and $2.0 \times 10^{29} \mathrm{~s}^{-1}$, respectively, and power-law exponents before $(-2.4)$ as after $(-2.6)$ perihelion and the value. Therefore there could be a moderate seasonal effect with values being nearly a factor of 1.7 times larger after perihelion, but the exponents are very similar. There are no permanent disruptive changes as in C/2001 A2 LINEAR. There is a small outburst seen in the image data on 2002 April 21, followed by a few days of falling-off by a factor of 2 compared with the trend. Dello Russo et al. (2004) report a power-law distribution based on 19 measurements of post-perihelion production rates from March 21 through April 13 , with a value at $1 \mathrm{AU}$ of $9.2 \times 10^{28} \mathrm{~s}^{-1}$ and a slope of -3.21 \pm 0.26 . This is consistent with both our fairly steep pre- and post-perihelion values $(-2.9$ and -2.6$)$.

While the volatile abundances in Ikeya-Zhang are rather similar to other bright Oort cloud comets such as Hale-Bopp and Hyakutake (Kawakita et al. 2003), it displays a somewhat steeper drop in production rate $(-2.6$ to -2.9$)$ as a function of heliocentric distance than the canonical $r^{-2}$ law.

Table 11 shows the deconvolved daily-average water production rates using the TRM and the results are plotted in Figure 6. There are a few noteworthy features in both the daily-averages
Table 8

Comet (LINEAR) WM1: Observational Circumstances and Single-Image Water Production Rates

\begin{tabular}{|c|c|c|c|c|c|}
\hline$\Delta T$ (days) & $(\mathrm{AU})$ & $\Delta(\mathrm{AU})$ & $g\left(\mathrm{~s}^{-1}\right)$ & $Q\left(\mathrm{~s}^{-1}\right)$ & $\Delta Q\left(\mathrm{~s}^{-}\right.$ \\
\hline-51.696 & 1.214 & 0.326 & .002452 & $6.372 \times 10^{28}$ & $6.075 \times 10^{25}$ \\
\hline-49.862 & 1.184 & 0.326 & 0.002451 & $6.797 \times 10^{28}$ & $6.073 \times 10^{24}$ \\
\hline-48.739 & 1.166 & & 0.002450 & $7.662 \times 10^{28}$ & $5.105 \times 10^{25}$ \\
\hline-47.856 & & & & $7.430 \times 10^{28}$ & $9.212 \times 10^{24}$ \\
\hline-46.866 & & & & $8.010 \times 10^{28}$ & $4.741 \times 10^{25}$ \\
\hline-45.476 & & & 0.002447 & $7.117 \times 10^{28}$ & $9.418 \times 10^{24}$ \\
\hline & & & & $8.248 \times 10^{28}$ & $4.732 \times 10^{25}$ \\
\hline-43 & & & 45 & $7.815 \times 10^{28}$ & $1.077 \times 10^{25}$ \\
\hline 033 & & & & $8.742 \times 10^{28}$ & $5.473 \times 10^{25}$ \\
\hline & & & & $7.389 \times 10^{28}$ & $1.261 \times 10^{25}$ \\
\hline & & & & $6.953 \times 10^{28}$ & $6.815 \times 10^{25}$ \\
\hline & 0.9 & & & $8.371 \times 10^{28}$ & $1.203 \times 10^{25}$ \\
\hline & & & & $7.997 \times 10^{28}$ & $5.846 \times 10^{25}$ \\
\hline & & & & $8.837 \times 10^{28}$ & $1.249 \times 10^{25}$ \\
\hline & & & & $9.220 \times 10^{28}$ & $6.626 \times 10^{25}$ \\
\hline & & & & $9.702 \times 10^{28}$ & $1.380 \times 10^{25}$ \\
\hline & 0.8 & 3 & 5 & $8.992 \times 10^{28}$ & $7.122 \times 10^{25}$ \\
\hline & & & & $9.248 \times 10^{28}$ & $1.478 \times 10^{25}$ \\
\hline & & & & $9.558 \times 10^{28}$ & $7.131 \times 10^{25}$ \\
\hline & 0 . & & & $1.203 \times 10^{29}$ & $1.489 \times 10^{25}$ \\
\hline & & & & $9.825 \times 10^{28}$ & $8.895 \times 10^{25}$ \\
\hline & & & & $1.120 \times 10^{29}$ & $1.705 \times 10^{25}$ \\
\hline-2 & 0.77 & & & $1.134 \times 10^{29}$ & $7.629 \times 10^{25}$ \\
\hline & & & & $1.055 \times 10^{29}$ & $1.865 \times 10^{25}$ \\
\hline & & & & $1.158 \times 10^{29}$ & $9.482 \times 10^{25}$ \\
\hline 437 & 0.708 & 0.725 & & $1.076 \times 10^{29}$ & $1.088 \times 10^{26}$ \\
\hline & & & & $1.274 \times 10^{29}$ & $2.301 \times 10^{25}$ \\
\hline & & & & $1.365 \times 10^{29}$ & $1.024 \times 10^{26}$ \\
\hline & 0.6 & & & $1.348 \times 10^{29}$ & $1.291 \times 10^{26}$ \\
\hline & & & & $1.674 \times 10^{29}$ & $2.333 \times 10^{25}$ \\
\hline & 0.6 & & & $1.651 \times 10^{29}$ & $1.136 \times 10^{26}$ \\
\hline & 0.60 & & & $1.977 \times 10^{29}$ & $2.385 \times 10^{25}$ \\
\hline & 0.6 & & & $2.137 \times 10^{29}$ & $1.064 \times 10^{26}$ \\
\hline & & & & $2.176 \times 10^{29}$ & $2.470 \times 10^{25}$ \\
\hline & & & & $2.295 \times 10^{29}$ & $1.202 \times 10^{26}$ \\
\hline & & & & $2.601 \times 10^{29}$ & $2.475 \times 10^{25}$ \\
\hline & & & & $2.337 \times 10^{29}$ & $9.806 \times 10^{25}$ \\
\hline-3.367 & 0.56 & 969 & & $2.754 \times 10^{29}$ & $1.002 \times 10^{28}$ \\
\hline & & & & $2.755 \times 10^{29}$ & $2.615 \times 10^{25}$ \\
\hline & & & & $3.201 \times 10^{29}$ & $1.148 \times 10^{26}$ \\
\hline & & 1.023 & & $3.082 \times 10^{29}$ & $2.495 \times 10^{25}$ \\
\hline & & & & $2.987 \times 10^{29}$ & $1.247 \times 10^{26}$ \\
\hline & & & & $3.062 \times 10^{29}$ & $2.498 \times 10^{25}$ \\
\hline 4.215 & 0.56 & & & $2.850 \times 10^{29}$ & $1.405 \times 10^{26}$ \\
\hline & & & & $3.736 \times 10^{29}$ & $8.082 \times 10^{25}$ \\
\hline & & & & $3.654 \times 10^{29}$ & $7.255 \times 10^{25}$ \\
\hline 27.015 & 0.817 & 1.210 & & $2.623 \times 10^{29}$ & $1.709 \times 10^{25}$ \\
\hline .405 & & & & $3.079 \times 10^{29}$ & $7.330 \times 10^{25}$ \\
\hline & & & & $3.020 \times 10^{29}$ & $7.960 \times 10^{25}$ \\
\hline & 0.88 & & & $2.741 \times 10^{29}$ & $1.610 \times 10^{25}$ \\
\hline .377 & 0.901 & & & $2.506 \times 10^{29}$ & $8.586 \times 10^{25}$ \\
\hline & & & & $2.097 \times 10^{29}$ & $8.847 \times 10^{25}$ \\
\hline & 0.95 & & & $1.951 \times 10^{29}$ & $2.081 \times 10^{25}$ \\
\hline .294 & 0.979 & 1.230 & 0.002212 & $1.700 \times 10^{29}$ & $1.061 \times 10^{26}$ \\
\hline & & & & $1.565 \times 10^{29}$ & $2.251 \times 10^{25}$ \\
\hline & 1.013 & 1.232 & & $1.491 \times 10^{29}$ & $9.702 \times 10^{25}$ \\
\hline & 1.062 & & 0.002207 & $1.424 \times 10^{29}$ & $8.533 \times 10^{25}$ \\
\hline & & & & $1.449 \times 10^{29}$ & $8.350 \times 10^{25}$ \\
\hline 45.485 & 1.113 & 1.236 & 0.002205 & $1.357 \times 10^{29}$ & $1.825 \times 10^{25}$ \\
\hline 46.379 & 1.127 & 1.236 & 0.002204 & $1.324 \times 10^{29}$ & $9.190 \times 10^{25}$ \\
\hline
\end{tabular}

Notes.

$\Delta T$ : time from perihelion 2002 January 22, in days for each SWAN image; $r$ : heliocentric distance (AU); $\Delta$ : geocentric distance (AU); $g$ : solar Lyman- $\alpha$ $g$-factor (photons $\mathrm{s}^{-1}$ ); $Q$ : water production rates for each image $\left(\mathrm{s}^{-1}\right) ; \Delta Q$ : $1 \sigma$ formal uncertainty $\left(\mathrm{s}^{-1}\right)$. 
Table 9

Comet (LINEAR) WM1: Daily-Averaged Deconvolved Water Production

\begin{tabular}{|c|c|c|}
\hline$\Delta T$ (days) & $Q\left(\mathrm{~s}^{-1}\right)$ & $\Delta Q\left(\mathrm{~s}^{-1}\right)$ \\
\hline-58.674 & $4.666 \times 10^{28}$ & $1.849 \times 10^{27}$ \\
\hline-57.674 & $4.499 \times 10^{28}$ & $4.283 \times 10^{26}$ \\
\hline-56.674 & $4.545 \times 10^{28}$ & $1.848 \times 10^{25}$ \\
\hline-55.674 & $5.381 \times 10^{28}$ & $4.169 \times 10^{26}$ \\
\hline-54.674 & $6.044 \times 10^{28}$ & $4.919 \times 10^{26}$ \\
\hline-53.674 & $4.242 \times 10^{28}$ & $3.737 \times 10^{26}$ \\
\hline-52.674 & $3.064 \times 10^{28}$ & $8.702 \times 10^{25}$ \\
\hline-51.674 & $3.154 \times 10^{28}$ & $3.401 \times 10^{25}$ \\
\hline-50.674 & $7.573 \times 10^{28}$ & $9.037 \times 10^{26}$ \\
\hline-49.674 & $9.246 \times 10^{28}$ & $1.378 \times 10^{27}$ \\
\hline-48.674 & $9.379 \times 10^{28}$ & $1.772 \times 10^{27}$ \\
\hline-47.674 & $7.111 \times 10^{28}$ & $1.091 \times 10^{27}$ \\
\hline-46.674 & $8.057 \times 10^{28}$ & $1.487 \times 10^{27}$ \\
\hline-45.674 & $8.795 \times 10^{28}$ & $1.817 \times 10^{26}$ \\
\hline-44.674 & $8.775 \times 10^{28}$ & $2.909 \times 10^{27}$ \\
\hline-43.674 & $9.299 \times 10^{28}$ & $2.351 \times 10^{27}$ \\
\hline-42.674 & $5.386 \times 10^{28}$ & $1.292 \times 10^{27}$ \\
\hline-41.674 & $6.432 \times 10^{28}$ & $6.138 \times 10^{26}$ \\
\hline-40.674 & $1.011 \times 10^{29}$ & $8.625 \times 10^{26}$ \\
\hline-39.674 & $6.458 \times 10^{28}$ & $6.814 \times 10^{26}$ \\
\hline-38.674 & $9.088 \times 10^{28}$ & $1.149 \times 10^{27}$ \\
\hline-37.674 & $6.612 \times 10^{28}$ & $8.647 \times 10^{26}$ \\
\hline-36.674 & $9.369 \times 10^{28}$ & $8.080 \times 10^{26}$ \\
\hline-35.674 & $7.916 \times 10^{28}$ & $2.907 \times 10^{27}$ \\
\hline-34.674 & $7.747 \times 10^{28}$ & $1.661 \times 10^{27}$ \\
\hline-33.674 & $1.114 \times 10^{29}$ & $4.091 \times 10^{27}$ \\
\hline-32.674 & $6.896 \times 10^{28}$ & $1.198 \times 10^{27}$ \\
\hline-31.674 & $7.660 \times 10^{28}$ & $1.061 \times 10^{27}$ \\
\hline-30.674 & $9.249 \times 10^{28}$ & $1.284 \times 10^{27}$ \\
\hline-29.674 & $1.350 \times 10^{29}$ & $5.915 \times 10^{27}$ \\
\hline-28.674 & $1.888 \times 10^{29}$ & $1.031 \times 10^{28}$ \\
\hline-27.674 & $8.907 \times 10^{28}$ & $1.757 \times 10^{27}$ \\
\hline-26.674 & $7.170 \times 10^{28}$ & $2.041 \times 10^{27}$ \\
\hline-25.674 & $7.218 \times 10^{28}$ & $4.521 \times 10^{26}$ \\
\hline-24.674 & $1.106 \times 10^{29}$ & $1.940 \times 10^{27}$ \\
\hline-23.674 & $8.769 \times 10^{28}$ & $4.443 \times 10^{27}$ \\
\hline-22.674 & $9.664 \times 10^{28}$ & $4.490 \times 10^{27}$ \\
\hline-21.674 & $8.389 \times 10^{28}$ & $4.654 \times 10^{27}$ \\
\hline-20.674 & $1.101 \times 10^{29}$ & $4.764 \times 10^{27}$ \\
\hline-19.674 & $1.402 \times 10^{29}$ & $6.820 \times 10^{27}$ \\
\hline-18.674 & $9.690 \times 10^{28}$ & $6.490 \times 10^{27}$ \\
\hline-17.674 & $8.929 \times 10^{28}$ & $1.928 \times 10^{27}$ \\
\hline-16.674 & $1.139 \times 10^{29}$ & $1.347 \times 10^{27}$ \\
\hline-15.674 & $1.475 \times 10^{29}$ & $6.051 \times 10^{27}$ \\
\hline-14.674 & $1.150 \times 10^{29}$ & $4.945 \times 10^{27}$ \\
\hline-13.674 & $1.494 \times 10^{29}$ & $3.009 \times 10^{27}$ \\
\hline-12.674 & $1.809 \times 10^{29}$ & $3.108 \times 10^{27}$ \\
\hline-11.674 & $1.419 \times 10^{29}$ & $6.489 \times 10^{27}$ \\
\hline-10.674 & $1.740 \times 10^{29}$ & $2.066 \times 10^{27}$ \\
\hline-9.674 & $1.605 \times 10^{29}$ & $5.345 \times 10^{27}$ \\
\hline-8.674 & $2.192 \times 10^{29}$ & $3.384 \times 10^{27}$ \\
\hline-7.674 & $2.170 \times 10^{29}$ & $3.515 \times 10^{27}$ \\
\hline-6.674 & $2.002 \times 10^{29}$ & $3.560 \times 10^{27}$ \\
\hline-5.674 & $2.082 \times 10^{29}$ & $6.762 \times 10^{27}$ \\
\hline-4.674 & $2.259 \times 10^{29}$ & $9.295 \times 10^{27}$ \\
\hline-3.674 & $2.131 \times 10^{29}$ & $3.809 \times 10^{27}$ \\
\hline-2.674 & $2.415 \times 10^{29}$ & $3.258 \times 10^{27}$ \\
\hline-1.674 & $2.626 \times 10^{29}$ & $2.041 \times 10^{27}$ \\
\hline-0.674 & $2.842 \times 10^{29}$ & $1.626 \times 10^{28}$ \\
\hline 0.326 & $2.407 \times 10^{29}$ & $1.344 \times 10^{29}$ \\
\hline 1.326 & $2.384 \times 10^{29}$ & $5.173 \times 10^{28}$ \\
\hline 13.326 & $4.233 \times 10^{29}$ & $2.845 \times 10^{29}$ \\
\hline 14.326 & $4.244 \times 10^{29}$ & $1.874 \times 10^{29}$ \\
\hline 15.326 & $4.208 \times 10^{29}$ & $1.661 \times 10^{29}$ \\
\hline 16.326 & $3.827 \times 10^{29}$ & $1.095 \times 10^{29}$ \\
\hline
\end{tabular}

Table 9

(Continued)

\begin{tabular}{lcc}
\hline \hline$\Delta T$ (days) & $Q\left(\mathrm{~s}^{-1}\right)$ & $\Delta Q\left(\mathrm{~s}^{-1}\right)$ \\
\hline 17.326 & $3.797 \times 10^{29}$ & $8.774 \times 10^{28}$ \\
18.326 & $3.657 \times 10^{29}$ & $8.068 \times 10^{28}$ \\
19.326 & $3.741 \times 10^{29}$ & $5.487 \times 10^{28}$ \\
20.326 & $3.108 \times 10^{29}$ & $5.783 \times 10^{28}$ \\
21.326 & $3.130 \times 10^{29}$ & $3.789 \times 10^{28}$ \\
22.326 & $3.179 \times 10^{29}$ & $1.908 \times 10^{28}$ \\
23.326 & $3.012 \times 10^{29}$ & $1.126 \times 10^{28}$ \\
24.326 & $2.944 \times 10^{29}$ & $7.499 \times 10^{28}$ \\
25.326 & $3.013 \times 10^{29}$ & $6.006 \times 10^{28}$ \\
26.326 & $2.993 \times 10^{29}$ & $5.447 \times 10^{28}$ \\
27.326 & $3.467 \times 10^{29}$ & $3.555 \times 10^{28}$ \\
28.326 & $3.523 \times 10^{29}$ & $7.294 \times 10^{28}$ \\
29.326 & $4.311 \times 10^{29}$ & $5.443 \times 10^{28}$ \\
30.326 & $2.172 \times 10^{29}$ & $2.074 \times 10^{28}$ \\
31.326 & $2.143 \times 10^{29}$ & $1.289 \times 10^{28}$ \\
32.326 & $2.364 \times 10^{29}$ & $2.467 \times 10^{28}$ \\
33.326 & $2.399 \times 10^{29}$ & $9.766 \times 10^{27}$ \\
34.326 & $1.959 \times 10^{29}$ & $3.606 \times 10^{28}$ \\
35.326 & $1.974 \times 10^{29}$ & $1.964 \times 10^{28}$ \\
36.326 & $1.733 \times 10^{29}$ & $4.520 \times 10^{28}$ \\
37.326 & $1.745 \times 10^{29}$ & $3.116 \times 10^{28}$ \\
38.326 & $1.513 \times 10^{29}$ & $3.359 \times 10^{28}$ \\
39.326 & $1.520 \times 10^{29}$ & $2.498 \times 10^{28}$ \\
40.326 & $1.596 \times 10^{29}$ & $3.344 \times 10^{28}$ \\
41.326 & $1.597 \times 10^{29}$ & $2.111 \times 10^{28}$ \\
42.326 & $1.632 \times 10^{29}$ & $3.717 \times 10^{28}$ \\
43.326 & $1.664 \times 10^{29}$ & $2.298 \times 10^{28}$ \\
\hline & &
\end{tabular}

Notes.

$\Delta T$ : time from perihelion 2002 January 22 , in days for each deconvolved value.

$Q$ : water production rates for each image $\left(\mathrm{s}^{-1}\right)$.

$\Delta Q: 1 \sigma$ formal uncertainty $\left(\mathrm{s}^{-1}\right)$.

and in a two-day-averaged version (not shown here). Right after perihelion the production rate drops by about $70 \%$ for 3 days before rising to the highest value about 7 days after perihelion. The daily-averaged deconvolved results show a fairly large outburst occurring from 42 to 44 days after perihelion. The twoday-averaged version shows a smaller rise covering a longer time period, so it appears to be real. However, this outburst is not seen in the SWAS results (Bensch \& Melnick 2006) covering the same time period. We have checked the original images just after this time period and there seems to be no interference from field stars that are normally excluded during the analysis process. There is another two-day long peak at 12 days before perihelion followed by a small dip in the production rate.

The comparison with other measures of the water production rates is fairly typical of the comparisons with the other comets. The SWAN values are similar to the SWAS (Bensch \& Melnick 2006) results near the beginning of the SWAS coverage at 35 days after perihelion (except for the peak at $\sim 43$ days mentioned above) but become gradually relatively higher toward the end of the observation period between 60 and 100 days after perihelion. As mentioned above, the infrared observations of water (Dello Russo et al. 2004) are somewhat lower than the SWAN observations. The ground-based $\mathrm{OH}$ result from Schleicher (reported by Irvine et al. 2003) is about a factor of 2 lower, possibly due to modeling differences. On the other hand, the agreement is quite good compared with the HST results of $\mathrm{OH}$ by Weaver et al. (2002) and with the radio observations by Biver et al. (2006) throughout the post-perihelion region 
Table 10

Comet Ikeya-Zhang: Observational Circumstances and Single-Image Water Production Rates

\begin{tabular}{|c|c|c|c|c|c|}
\hline$\Delta T$ (days) & $r(\mathrm{AU})$ & $\Delta(\mathrm{AU})$ & $g\left(\mathrm{~s}^{-1}\right)$ & $Q\left(\mathrm{~s}^{-1}\right)$ & $\Delta Q\left(\mathrm{~s}^{-1}\right)$ \\
\hline-44.542 & 1.094 & 1.540 & 0.002433 & $1.288 \times 10^{29}$ & $1.859 \times 10^{26}$ \\
\hline-38.049 & 0.984 & 1.461 & 0.002428 & $1.244 \times 10^{29}$ & $1.463 \times 10^{26}$ \\
\hline-36.995 & 0.966 & 1.448 & 0.002427 & $1.817 \times 10^{29}$ & $1.190 \times 10^{26}$ \\
\hline-35.871 & 0.947 & 1.433 & 0.002425 & $1.348 \times 10^{29}$ & $1.347 \times 10^{26}$ \\
\hline-33.496 & 0.907 & 1.400 & 0.002423 & $1.472 \times 10^{29}$ & $1.286 \times 10^{26}$ \\
\hline-32.174 & 0.885 & 1.382 & 0.002422 & $1.447 \times 10^{29}$ & $1.236 \times 10^{26}$ \\
\hline-27.465 & 0.807 & 1.312 & 0.002387 & $2.080 \times 10^{29}$ & $8.899 \times 10^{25}$ \\
\hline-25.576 & 0.776 & 1.282 & 0.002355 & $2.489 \times 10^{29}$ & $6.101 \times 10^{25}$ \\
\hline-23.507 & 0.743 & 1.249 & 0.002353 & $3.383 \times 10^{29}$ & $5.382 \times 10^{25}$ \\
\hline-20.508 & 0.696 & 1.198 & 0.002321 & $2.796 \times 10^{29}$ & $5.435 \times 10^{25}$ \\
\hline-18.605 & 0.668 & 1.165 & 0.002290 & $3.796 \times 10^{29}$ & $4.870 \times 10^{25}$ \\
\hline-16.550 & 0.639 & 1.128 & 0.002234 & $4.531 \times 10^{29}$ & $4.335 \times 10^{25}$ \\
\hline-13.522 & 0.600 & 1.072 & 0.002185 & $5.522 \times 10^{29}$ & $4.225 \times 10^{25}$ \\
\hline-11.466 & 0.576 & 1.033 & 0.002141 & $6.921 \times 10^{29}$ & $3.963 \times 10^{25}$ \\
\hline-9.549 & 0.556 & 0.996 & 0.002083 & $7.778 \times 10^{29}$ & $3.994 \times 10^{25}$ \\
\hline-6.536 & 0.531 & 0.936 & 0.002016 & $6.494 \times 10^{29}$ & $4.352 \times 10^{25}$ \\
\hline-4.480 & 0.518 & 0.895 & 0.001970 & $8.719 \times 10^{29}$ & $4.153 \times 10^{25}$ \\
\hline-2.564 & 0.511 & 0.857 & 0.001943 & $1.116 \times 10^{30}$ & $3.592 \times 10^{25}$ \\
\hline 0.441 & 0.507 & 0.799 & 0.001914 & $9.439 \times 10^{29}$ & $3.689 \times 10^{25}$ \\
\hline 2.521 & 0.511 & 0.759 & 0.001906 & $1.104 \times 10^{30}$ & $3.008 \times 10^{25}$ \\
\hline 4.442 & 0.518 & 0.725 & 0.001913 & $8.403 \times 10^{29}$ & $3.265 \times 10^{25}$ \\
\hline 7.494 & 0.538 & 0.672 & 0.001951 & $6.310 \times 10^{29}$ & $2.927 \times 10^{25}$ \\
\hline 8.554 & 0.547 & 0.655 & 0.001974 & $9.014 \times 10^{29}$ & $2.604 \times 10^{25}$ \\
\hline 10.467 & 0.565 & 0.626 & 0.001998 & $8.873 \times 10^{29}$ & $2.365 \times 10^{25}$ \\
\hline 12.360 & 0.586 & 0.599 & 0.002039 & $9.619 \times 10^{29}$ & $2.026 \times 10^{25}$ \\
\hline 15.500 & 0.625 & 0.558 & 0.002088 & $7.330 \times 10^{29}$ & $1.915 \times 10^{25}$ \\
\hline 17.417 & 0.651 & 0.536 & 0.002105 & $5.576 \times 10^{29}$ & $2.024 \times 10^{25}$ \\
\hline 19.332 & 0.679 & 0.516 & 0.002122 & $5.272 \times 10^{29}$ & $1.752 \times 10^{25}$ \\
\hline 22.361 & 0.725 & 0.487 & 0.002139 & $5.018 \times 10^{29}$ & $1.519 \times 10^{25}$ \\
\hline 24.279 & 0.755 & 0.471 & 0.002157 & $4.792 \times 10^{29}$ & $1.462 \times 10^{25}$ \\
\hline 26.169 & 0.786 & 0.457 & 0.002175 & $4.076 \times 10^{29}$ & $1.364 \times 10^{25}$ \\
\hline 29.719 & 0.844 & 0.434 & 0.002172 & $3.394 \times 10^{29}$ & $1.163 \times 10^{25}$ \\
\hline 31.637 & 0.876 & 0.425 & 0.002191 & $2.946 \times 10^{29}$ & $1.152 \times 10^{25}$ \\
\hline 33.526 & 0.908 & 0.417 & 0.002189 & $3.011 \times 10^{29}$ & $1.171 \times 10^{25}$ \\
\hline 34.450 & 0.923 & 0.414 & 0.002189 & $3.844 \times 10^{29}$ & $9.336 \times 10^{25}$ \\
\hline 36.422 & 0.957 & 0.409 & 0.002187 & $1.896 \times 10^{29}$ & $1.240 \times 10^{25}$ \\
\hline 38.492 & 0.992 & 0.405 & 0.002185 & $1.634 \times 10^{29}$ & $1.132 \times 10^{25}$ \\
\hline 40.332 & 1.023 & 0.404 & 0.002183 & $1.656 \times 10^{29}$ & $9.248 \times 10^{24}$ \\
\hline 43.342 & 1.074 & 0.405 & 0.002181 & $1.741 \times 10^{29}$ & $5.705 \times 10^{24}$ \\
\hline 45.151 & 1.104 & 0.409 & 0.002179 & $1.742 \times 10^{29}$ & $6.604 \times 10^{24}$ \\
\hline 46.847 & 1.133 & 0.414 & 0.002178 & $1.634 \times 10^{29}$ & $9.110 \times 10^{24}$ \\
\hline 50.152 & 1.189 & 0.427 & 0.002175 & $1.323 \times 10^{29}$ & $1.526 \times 10^{25}$ \\
\hline 52.180 & 1.222 & 0.439 & 0.002152 & $1.342 \times 10^{29}$ & $1.689 \times 10^{25}$ \\
\hline 53.242 & 1.240 & 0.446 & 0.002152 & $1.244 \times 10^{29}$ & $1.955 \times 10^{25}$ \\
\hline 55.630 & 1.280 & 0.464 & 0.002150 & $1.067 \times 10^{29}$ & $2.676 \times 10^{25}$ \\
\hline 58.602 & 1.329 & 0.490 & 0.002147 & $9.149 \times 10^{28}$ & $3.067 \times 10^{25}$ \\
\hline 60.628 & 1.362 & 0.510 & 0.002146 & $9.920 \times 10^{28}$ & $3.238 \times 10^{25}$ \\
\hline 63.628 & 1.411 & 0.543 & 0.002143 & $9.064 \times 10^{28}$ & $3.967 \times 10^{25}$ \\
\hline 65.680 & 1.444 & 0.568 & 0.002122 & $8.984 \times 10^{28}$ & $4.464 \times 10^{25}$ \\
\hline 67.599 & 1.475 & 0.593 & 0.002120 & $8.045 \times 10^{28}$ & $5.151 \times 10^{25}$ \\
\hline 71.039 & 1.530 & 0.641 & 0.002117 & $6.762 \times 10^{28}$ & $6.714 \times 10^{25}$ \\
\hline 73.091 & 1.563 & 0.671 & 0.002115 & $7.366 \times 10^{28}$ & $6.741 \times 10^{25}$ \\
\hline 74.789 & 1.590 & 0.697 & 0.002114 & $6.742 \times 10^{28}$ & $8.050 \times 10^{25}$ \\
\hline 77.605 & 1.634 & 0.743 & 0.002112 & $6.818 \times 10^{28}$ & $7.141 \times 10^{25}$ \\
\hline 79.639 & 1.666 & 0.777 & 0.002091 & $5.748 \times 10^{28}$ & $1.004 \times 10^{26}$ \\
\hline 81.689 & 1.697 & 0.813 & 0.002089 & $5.150 \times 10^{28}$ & $1.096 \times 10^{26}$ \\
\hline 84.689 & 1.744 & 0.867 & 0.002086 & $4.426 \times 10^{28}$ & $1.320 \times 10^{26}$ \\
\hline 91.438 & 1.846 & 0.997 & 0.002080 & $3.457 \times 10^{28}$ & $2.045 \times 10^{26}$ \\
\hline 93.138 & 1.872 & 1.031 & 0.002060 & $3.292 \times 10^{28}$ & $2.012 \times 10^{26}$ \\
\hline 107.164 & 2.078 & 1.334 & 0.002048 & $2.722 \times 10^{28}$ & $1.062 \times 10^{25}$ \\
\hline 113.498 & 2.170 & 1.481 & 0.002025 & $2.373 \times 10^{28}$ & $1.380 \times 10^{25}$ \\
\hline 119.461 & 2.254 & 1.624 & 0.002020 & $1.776 \times 10^{28}$ & $6.547 \times 10^{26}$ \\
\hline
\end{tabular}

Notes.

$\Delta T$ : time from perihelion 2002 March 18, in days for each SWAN image; $r$ : heliocentric distance (AU); $\Delta$ : geocentric distance (AU); $g$ : solar Lyman- $\alpha$ gfactor (photons $\mathrm{s}^{-1}$ ); $Q$ : water production rates for each image $\left(\mathrm{s}^{-1}\right) ; \Delta Q: 1 \sigma$ formal uncertainty $\left(\mathrm{s}^{-1}\right)$.
Table 11

Comet Ikeya-Zhang: Daily-Averaged Deconvolved Water Production Rates

\begin{tabular}{|c|c|c|}
\hline$\Delta T$ (days) & $Q\left(\mathrm{~s}^{-1}\right)$ & $\Delta Q\left(\mathrm{~s}^{-1}\right)$ \\
\hline-43.980 & $1.108 \times 10^{29}$ & $3.002 \times 10^{28}$ \\
\hline-42.980 & $1.159 \times 10^{29}$ & $3.143 \times 10^{28}$ \\
\hline-41.980 & $1.096 \times 10^{29}$ & $1.505 \times 10^{28}$ \\
\hline-40.980 & $1.327 \times 10^{29}$ & $2.968 \times 10^{28}$ \\
\hline-39.980 & $1.474 \times 10^{29}$ & $1.984 \times 10^{28}$ \\
\hline-38.980 & $1.181 \times 10^{29}$ & $3.536 \times 10^{28}$ \\
\hline-37.980 & $1.152 \times 10^{29}$ & $2.751 \times 10^{28}$ \\
\hline-36.980 & $1.337 \times 10^{29}$ & $2.534 \times 10^{28}$ \\
\hline-35.980 & $1.345 \times 10^{29}$ & $1.247 \times 10^{28}$ \\
\hline-34.980 & $1.492 \times 10^{29}$ & $4.810 \times 10^{28}$ \\
\hline-33.980 & $1.488 \times 10^{29}$ & $4.077 \times 10^{28}$ \\
\hline-32.980 & $1.640 \times 10^{29}$ & $4.153 \times 10^{28}$ \\
\hline-31.980 & $1.643 \times 10^{29}$ & $3.543 \times 10^{28}$ \\
\hline-30.980 & $1.988 \times 10^{29}$ & $3.588 \times 10^{28}$ \\
\hline-29.980 & $2.072 \times 10^{29}$ & $2.149 \times 10^{28}$ \\
\hline-28.980 & $2.397 \times 10^{29}$ & $4.358 \times 10^{28}$ \\
\hline-27.980 & $2.645 \times 10^{29}$ & $2.645 \times 10^{28}$ \\
\hline-26.980 & $2.538 \times 10^{29}$ & $6.449 \times 10^{28}$ \\
\hline-25.980 & $3.035 \times 10^{29}$ & $4.336 \times 10^{28}$ \\
\hline-24.980 & $2.350 \times 10^{29}$ & $6.835 \times 10^{27}$ \\
\hline-23.980 & $2.350 \times 10^{29}$ & $6.784 \times 10^{27}$ \\
\hline-22.980 & $2.977 \times 10^{29}$ & $3.723 \times 10^{28}$ \\
\hline-21.980 & $2.974 \times 10^{29}$ & $2.743 \times 10^{28}$ \\
\hline-20.980 & $3.663 \times 10^{29}$ & $4.567 \times 10^{28}$ \\
\hline-19.980 & $3.852 \times 10^{29}$ & $1.472 \times 10^{28}$ \\
\hline-18.980 & $3.626 \times 10^{29}$ & $3.850 \times 10^{28}$ \\
\hline-17.980 & $3.661 \times 10^{29}$ & $2.886 \times 10^{28}$ \\
\hline-16.980 & $4.092 \times 10^{29}$ & $3.423 \times 10^{28}$ \\
\hline-15.980 & $4.351 \times 10^{29}$ & $2.038 \times 10^{28}$ \\
\hline-14.980 & $4.992 \times 10^{29}$ & $2.801 \times 10^{28}$ \\
\hline-13.980 & $5.693 \times 10^{29}$ & $1.605 \times 10^{28}$ \\
\hline-12.980 & $5.742 \times 10^{29}$ & $4.952 \times 10^{27}$ \\
\hline-11.980 & $8.660 \times 10^{29}$ & $5.825 \times 10^{28}$ \\
\hline-10.980 & $3.790 \times 10^{29}$ & $2.251 \times 10^{28}$ \\
\hline-9.980 & $4.583 \times 10^{29}$ & $2.350 \times 10^{28}$ \\
\hline-8.980 & $6.039 \times 10^{29}$ & $3.455 \times 10^{28}$ \\
\hline-7.980 & $7.128 \times 10^{29}$ & $4.878 \times 10^{28}$ \\
\hline-6.980 & $6.569 \times 10^{29}$ & $1.822 \times 10^{28}$ \\
\hline-5.980 & $7.288 \times 10^{29}$ & $4.278 \times 10^{28}$ \\
\hline-4.980 & $9.284 \times 10^{29}$ & $4.211 \times 10^{28}$ \\
\hline-3.980 & $8.153 \times 10^{29}$ & $5.350 \times 10^{28}$ \\
\hline-2.980 & $7.286 \times 10^{29}$ & $1.699 \times 10^{28}$ \\
\hline-1.980 & $6.988 \times 10^{29}$ & $9.410 \times 10^{27}$ \\
\hline-0.980 & $8.156 \times 10^{29}$ & $5.546 \times 10^{27}$ \\
\hline 0.020 & $1.129 \times 10^{30}$ & $6.669 \times 10^{27}$ \\
\hline 1.020 & $6.477 \times 10^{29}$ & $5.445 \times 10^{27}$ \\
\hline 2.020 & $7.560 \times 10^{29}$ & $2.290 \times 10^{28}$ \\
\hline 3.020 & $5.533 \times 10^{29}$ & $2.044 \times 10^{28}$ \\
\hline 4.020 & $5.696 \times 10^{29}$ & $6.575 \times 10^{27}$ \\
\hline 5.020 & $5.970 \times 10^{29}$ & $4.060 \times 10^{27}$ \\
\hline 6.020 & $7.847 \times 10^{29}$ & $6.035 \times 10^{27}$ \\
\hline 7.020 & $1.332 \times 10^{30}$ & $6.909 \times 10^{27}$ \\
\hline 8.020 & $9.316 \times 10^{29}$ & $1.982 \times 10^{28}$ \\
\hline 9.020 & $8.967 \times 10^{29}$ & $2.072 \times 10^{28}$ \\
\hline 10.020 & $8.864 \times 10^{29}$ & $8.830 \times 10^{27}$ \\
\hline 11.020 & $8.325 \times 10^{29}$ & $6.930 \times 10^{28}$ \\
\hline 12.020 & $6.758 \times 10^{29}$ & $1.401 \times 10^{28}$ \\
\hline 13.020 & $6.075 \times 10^{29}$ & $7.597 \times 10^{27}$ \\
\hline 14.020 & $4.688 \times 10^{29}$ & $9.535 \times 10^{27}$ \\
\hline 15.020 & $4.701 \times 10^{29}$ & $5.706 \times 10^{27}$ \\
\hline 16.020 & $4.369 \times 10^{29}$ & $5.474 \times 10^{27}$ \\
\hline 17.020 & $5.779 \times 10^{29}$ & $3.285 \times 10^{27}$ \\
\hline 18.020 & $4.031 \times 10^{29}$ & $1.579 \times 10^{28}$ \\
\hline 19.020 & $4.081 \times 10^{29}$ & $5.575 \times 10^{27}$ \\
\hline 20.020 & $5.494 \times 10^{29}$ & $5.674 \times 10^{27}$ \\
\hline 21.020 & $4.336 \times 10^{29}$ & $1.157 \times 10^{28}$ \\
\hline 22.020 & $5.408 \times 10^{29}$ & $8.234 \times 10^{27}$ \\
\hline & $3.522 \times 10^{29}$ & $6.490 \times 10^{27}$ \\
\hline
\end{tabular}


Table 11

(Continued)

\begin{tabular}{|c|c|c|}
\hline$\Delta T$ (days) & $Q\left(\mathrm{~s}^{-1}\right)$ & $\Delta Q\left(\mathrm{~s}^{-1}\right)$ \\
\hline 24.020 & $5.135 \times 10^{29}$ & $4.117 \times 10^{27}$ \\
\hline 25.020 & $2.736 \times 10^{29}$ & $5.843 \times 10^{27}$ \\
\hline 26.020 & $2.748 \times 10^{29}$ & $2.837 \times 10^{27}$ \\
\hline 27.020 & $3.683 \times 10^{29}$ & $2.661 \times 10^{27}$ \\
\hline 28.020 & $6.777 \times 10^{29}$ & $3.334 \times 10^{27}$ \\
\hline 29.020 & $2.963 \times 10^{29}$ & $1.077 \times 10^{28}$ \\
\hline 30.020 & $3.919 \times 10^{29}$ & $1.699 \times 10^{28}$ \\
\hline 31.020 & $2.930 \times 10^{29}$ & $1.582 \times 10^{28}$ \\
\hline 32.020 & $3.223 \times 10^{29}$ & $1.543 \times 10^{28}$ \\
\hline 33.020 & $1.858 \times 10^{29}$ & $9.688 \times 10^{27}$ \\
\hline 34.020 & $1.721 \times 10^{29}$ & $5.947 \times 10^{27}$ \\
\hline 35.020 & $1.692 \times 10^{29}$ & $1.646 \times 10^{28}$ \\
\hline 36.020 & $1.688 \times 10^{29}$ & $5.373 \times 10^{27}$ \\
\hline 37.020 & $1.466 \times 10^{29}$ & $2.752 \times 10^{27}$ \\
\hline 38.020 & $1.960 \times 10^{29}$ & $1.003 \times 10^{27}$ \\
\hline 39.020 & $2.210 \times 10^{29}$ & $3.948 \times 10^{27}$ \\
\hline 40.020 & $2.084 \times 10^{29}$ & $7.059 \times 10^{27}$ \\
\hline 41.020 & $1.478 \times 10^{29}$ & $1.456 \times 10^{27}$ \\
\hline 42.020 & $5.288 \times 10^{29}$ & $2.096 \times 10^{28}$ \\
\hline 43.020 & $5.745 \times 10^{29}$ & $1.927 \times 10^{27}$ \\
\hline 44.020 & $1.915 \times 10^{29}$ & $7.179 \times 10^{28}$ \\
\hline 45.020 & $1.685 \times 10^{29}$ & $4.132 \times 10^{28}$ \\
\hline 46.020 & $1.284 \times 10^{29}$ & $1.025 \times 10^{28}$ \\
\hline 47.020 & $1.342 \times 10^{29}$ & $4.041 \times 10^{27}$ \\
\hline 48.020 & $1.705 \times 10^{29}$ & $6.818 \times 10^{27}$ \\
\hline 49.020 & $1.392 \times 10^{29}$ & $6.406 \times 10^{27}$ \\
\hline 50.020 & $1.630 \times 10^{29}$ & $7.417 \times 10^{27}$ \\
\hline 51.020 & $1.290 \times 10^{29}$ & $7.491 \times 10^{27}$ \\
\hline 52.020 & $1.162 \times 10^{29}$ & $1.020 \times 10^{28}$ \\
\hline 53.020 & $1.228 \times 10^{29}$ & $8.371 \times 10^{27}$ \\
\hline 54.020 & $1.109 \times 10^{29}$ & $4.816 \times 10^{26}$ \\
\hline 55.020 & $1.046 \times 10^{29}$ & $7.066 \times 10^{27}$ \\
\hline 56.020 & $1.216 \times 10^{29}$ & $6.416 \times 10^{27}$ \\
\hline 57.020 & $1.486 \times 10^{29}$ & $3.231 \times 10^{27}$ \\
\hline 58.020 & $1.053 \times 10^{29}$ & $1.155 \times 10^{28}$ \\
\hline 59.020 & $1.075 \times 10^{29}$ & $7.536 \times 10^{27}$ \\
\hline 60.020 & $1.008 \times 10^{29}$ & $6.815 \times 10^{27}$ \\
\hline 61.020 & $1.113 \times 10^{29}$ & $5.591 \times 10^{27}$ \\
\hline 62.020 & $1.290 \times 10^{29}$ & $2.002 \times 10^{27}$ \\
\hline 63.020 & $1.089 \times 10^{29}$ & $8.727 \times 10^{27}$ \\
\hline 64.020 & $1.220 \times 10^{29}$ & $3.327 \times 10^{27}$ \\
\hline 65.020 & $9.761 \times 10^{28}$ & $9.247 \times 10^{27}$ \\
\hline 66.020 & $8.111 \times 10^{28}$ & $1.597 \times 10^{28}$ \\
\hline 67.020 & $7.853 \times 10^{28}$ & $1.159 \times 10^{28}$ \\
\hline 68.020 & $7.805 \times 10^{28}$ & $8.575 \times 10^{27}$ \\
\hline 69.020 & $7.980 \times 10^{28}$ & $5.342 \times 10^{27}$ \\
\hline 70.020 & $8.380 \times 10^{28}$ & $9.266 \times 10^{27}$ \\
\hline 71.020 & $8.825 \times 10^{28}$ & $5.649 \times 10^{27}$ \\
\hline 72.020 & $8.631 \times 10^{28}$ & $6.771 \times 10^{27}$ \\
\hline 73.020 & $7.427 \times 10^{28}$ & $1.150 \times 10^{28}$ \\
\hline 74.020 & $7.364 \times 10^{28}$ & $7.575 \times 10^{27}$ \\
\hline 75.020 & $7.484 \times 10^{28}$ & $3.613 \times 10^{27}$ \\
\hline 76.020 & $6.817 \times 10^{28}$ & $1.122 \times 10^{28}$ \\
\hline 77.020 & $7.025 \times 10^{28}$ & $5.558 \times 10^{27}$ \\
\hline 78.020 & $6.449 \times 10^{28}$ & $1.283 \times 10^{28}$ \\
\hline 79.020 & $6.632 \times 10^{28}$ & $4.833 \times 10^{27}$ \\
\hline 80.020 & $5.516 \times 10^{28}$ & $1.552 \times 10^{28}$ \\
\hline 81.020 & $5.486 \times 10^{28}$ & $9.476 \times 10^{27}$ \\
\hline 82.020 & $5.635 \times 10^{28}$ & $4.693 \times 10^{27}$ \\
\hline 83.020 & $4.944 \times 10^{28}$ & $2.184 \times 10^{28}$ \\
\hline 84.020 & $4.722 \times 10^{28}$ & $1.923 \times 10^{28}$ \\
\hline 85.020 & $4.846 \times 10^{28}$ & $1.746 \times 10^{29}$ \\
\hline 86.020 & $4.768 \times 10^{28}$ & $1.101 \times 10^{29}$ \\
\hline 87.020 & $4.667 \times 10^{28}$ & $8.941 \times 10^{28}$ \\
\hline 88.020 & $4.719 \times 10^{28}$ & $5.801 \times 10^{28}$ \\
\hline 89.020 & $4.897 \times 10^{28}$ & $1.927 \times 10^{28}$ \\
\hline
\end{tabular}

Notes. $\Delta T$ : time from perihelion 2002 March 18, in days for each deconvolved value; $Q$ : water production rates for each image $\left(\mathrm{s}^{-1}\right) ; \Delta Q: 1 \sigma$ formal uncertainty $\left(\mathrm{s}^{-1}\right)$.

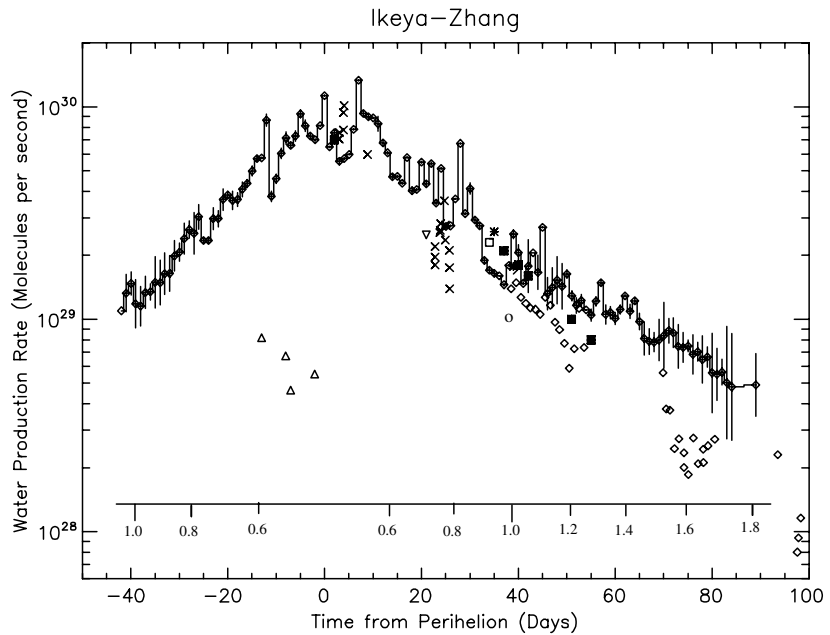

Figure 6. Daily-averaged water production rates of comet P153/Ikeya-Zhang. See Figure 2 caption for details. The other water production rate values are: isolated diamonds, Bensch et al. (2004); filled squares, Biver et al. (2006); ×, Bonev/Mumma/Dello Russo; open circle, Hatchell et al. (2005); asterisk, Lecacheux et al. (2003); open square, Weaver et al. (2002); downward-pointing triangle, Schleicher. The inset scale gives the heliocentric distance in AU.

where they overlap and also with their one observation two days after perihelion. Finally, the $\mathrm{OH}$ radio observations by Lovell et al. (2008) are again systematically quite low compared with the SWAN results and the near-perihelion result of Biver et al. (2006).

\section{DISCUSSION}

As found in a number of previous studies (Smyth et al. 1995; Combi et al. 1998, 2000, 2005), water production rates derived from observations of $\mathrm{H} \mathrm{Ly}-\alpha$ on the whole tend to give similar to somewhat larger values on the average than other methods (space-based $\mathrm{OH}$, ground-based $\mathrm{OH}$, radio $\mathrm{OH}$, water infrared, and water submillimeter). This trend generally holds for the five comets reported here; however more detailed comparisons show there are certainly times when there is quite consistent agreement with each of the other techniques, making any broad characterizations perhaps less universally true than previously thought. The exception is the ground-based $\mathrm{OH}$ observations that are calculated with the Haser model parameters given in the 85 comets survey of A'Hearn et al. (1995) and that give values generally lower than most of the others, most of the time as explained in the case for Halley (Schleicher et al. 1998). While there remains some variation in the absolute level of water production rates between emissions and measurement techniques, generally the comparisons of variation of water production rates among the methods are fairly consistent.

Studies of the compositions of Oort cloud comets (Mumma et al. 2003; Biver et al. 2006; Bockelée-Morvan et al. 2004) find diversity in volatile abundances. The SWAN observations of similar comets indicate that there also seems to be a wide range of water production variation with heliocentric distance. One advantage of the SWAN camera is that it can observe $\mathrm{H}$ Ly- $\alpha$ in most comets over large portions of their active apparitions whether they are northern or southern hemisphere objects and for extended and continuous periods before and after perihelion. Therefore, we can establish a fairly consistent set of water production "light curves" that are averaged over longer portions of their orbit than other observations that can have spottier coverage. 
Table 12

Volatile Composition for Three Water Variation Groups

\begin{tabular}{lcccc}
\hline \hline Comet & $Q_{1}\left(\mathrm{H}_{2} \mathrm{O}\right)^{\mathrm{a}}$ & $p^{\mathrm{b}}$ & $\mathrm{CO} / \mathrm{H}_{2} \mathrm{O}(\%)$ & $\mathrm{C}_{2} \mathrm{H}_{6} / \mathrm{H}_{2} \mathrm{O}(\%)$ \\
\hline \multicolumn{5}{c}{ Steep slope } \\
C 1999 T1 McNaught-Hartley & $2.9 \times 10^{29}$ & -3.3 & 15 & 0.5 \\
C 2001 A2 LINEAR & $8.2 \times 10^{28}$ & -4.5 & 1.5 & 1.6 \\
& \multicolumn{5}{c}{ Moderate slope } \\
C 1995 OI Hale-Bopp & $1.3 \times 10^{31}-2.6$ & 23 & 0.6 \\
C 1999 H1 Lee & $1.5 \times 10^{29}$ & -2.7 & $2-4$ & 0.7 \\
P153/Ikeya-Zhang & $1.8 \times 10^{29}$ & -2.7 & 4 & 0.6 \\
C 1996 B2 Hyakutake & $2.7 \times 10^{29}$ & -2.1 & $19-30$ & 0.6 \\
& \multicolumn{5}{c}{ Shallow slope } & & 0.1 \\
C 1999 S4 LINEAR & $1.7 \times 10^{28}$ & -1.6 & 0.6 & 0.5 \\
C 2001 WM1 LINEAR & $1.1 \times 10^{29}$ & -1.4 & 1 & \\
\hline
\end{tabular}

Notes.

a Average pre-post water production rate at $1 \mathrm{AU}$.

${ }^{\mathrm{b}}$ Average pre-post power-law exponent.

In cases where there are pre- to post-perihelion differences in the activity, the pre- and post- perihelion power-law slopes for the same comet are similar to one another. Therefore, there are real differences in the rate of sublimation from comet to comet that seem to have more to do with the physical make up of the comet than simply shape or broad heterogeneity of volatile distribution around the surface. There are, though, clear variations that are probably due to seasonal effects owing to nucleus shape or distribution of active areas on the surface, but these are superimposed over the general heliocentric distance variation. In such cases though there are real differences in slope between comets, but different areas on or near the surfaces of the same comet that contribute to the overall progression of water sublimation with changing heliocentric distance seem to be the same in that sense with seasonal differences superimposed. One exception to this might be A2 that underwent a large outburst near the beginning of the first SWAN observations, accompanied by the release of a major fragment, and that was followed by a steep increase in production rate throughout the preperihelion leg and a flatter slope after perihelion. Clearly, this large outburst that was followed by a large increase in activity changed the nucleus in a permanent and dramatic way. Nonetheless, the slopes both before and after perihelion were quite steep.

A number of outburst events were detected in this set of comets. Outbursts in A2 were clearly the most numerous and were reported extensively by other observers. Some of the outbursts were accompanied by the release of fragments. The continuous coverage of water production rate by the SWAN observations was able to show the outbursts responsible for the release of fragments also seen easily in the visible photometry and other observations. The initiation of the outburst seen in the deconvolved water production rates either coincided or slightly preceded that seen in the visible photometry. The delay of the photometry could be due to the rather fast propagation of the gas compared with the dust for this level of production rate comet. Although the SWAN FOV is large and it takes time to produce $\mathrm{H}$ atoms from water and $\mathrm{OH}$, the TRM deconvolution process accounts for all the lag times associated with the photodissociation lifetimes of water and $\mathrm{OH}$ as well as the propagation times of the entire chain into the coma.

If we were to classify the admittedly small group of eight Oort cloud comets observed extensively by SWAN by adding the previously reported results from C/1995 O1 Hale-Bopp (Combi et al. 2000) as well as a recent, more complete reanalysis
(Combi et al. 2006), C/1996 B2 Hyakutake (Combi et al. 2005) and C/1999 S4 LINEAR (Mäkinen et al. 2001) (hereafter S4) to the five reported for the first time here, there are three natural groupings given the slopes of their heliocentric distance variations. Hale-Bopp, Lee, I-Z, and Hyakutake make up the normal group with slopes in the range of $r^{-2}$ to $r^{-3}$. Based on the extensive reported observations, 1P/Halley would probably be a member of this group. There are two comets with steep slopes, namely $\mathrm{M}-\mathrm{H}$ and $\mathrm{A} 2$, and two with very shallow slopes, S4 and WM1. The classification as well as the reported CO and ethane abundances relative to water are summarized in Table 12

In this limited set of eight comets there is no obvious consistent association of volatile composition or of absolute production rate level with the value of the slope. Hale-Bopp and Hyakutake were relatively $\mathrm{CO}$ rich with variation in the moderate, $r^{-2}-r^{-3}$, group, while Lee and I-Z have somewhat depleted $(2-4 \%) \mathrm{CO}$ abundances. $\mathrm{M}-\mathrm{H}$ has a high (15\%) $\mathrm{CO}$ abundance and the steepest slope. Based on both the shallow slope and the low CO abundance WM1 might otherwise seem to be a larger version of S4, the comet that completely disintegrated after a number of outburst events. Mumma et al. (2001a) suggested that S4 might have formed closer to the inner solar system (5-10 AU) rather than beyond $30 \mathrm{AU}$, where many others are believed to have formed. But one interesting difference between WM1 and S4 is that the relative abundances of the supervolatile ethane are not the same. Whereas the ethane abundance in S4 was also very low $(0.1 \%)$, that in WM1 was $0.5 \%$, putting ethane the nominal group. This could be consistent with Lara et al. (2004b) having reported that WM1 was also a $\mathrm{C}_{2}$-rich comet if in fact $\mathrm{C}_{2}$ is associated with hydrocarbons such as acetylene and ethane (Helbert et al. 2005). However, acetylene, which is the more dominant producer of $\mathrm{C}_{2}$, has not been reported in WM1. Since the comet is $\mathrm{C}_{2}$ rich, this could mean that the comet is acetylene rich despite having a normal amount of ethane. The water production rate of WM1 was nearly an order of magnitude larger than S4 at comparable heliocentric distances. WM1 displayed two outbursts but certainly not the numerous outbursts and fragmentation events of S4, nor its catastrophic disintegration. The association of S4 and WM1 raises the question: if $\mathrm{CO}$ is underabundant either because of the formation location, or because of subsequent depletion by evolution, why is ethane quite abundant in WM1 but depleted in S4? Perhaps it says something about the intimate compositional mixing of different molecules in ices themselves, and where 
and when the different volatiles condensed. It is also possible that we still suffer from small number sampling both regarding volatile composition and consideration of their variation of production rate with heliocentric distance. Perhaps with time and an increase in the sample of comets we will have detailed volatile composition, measured production rate levels and their variations with heliocentric distance.

It appears that the overall slope of the production rate variation with heliocentric distance is yet another important property of comets that should be considered in characterizing them. Within this group of comets the slope is not obviously correlated with the absolute level of the production rate or with the volatility as measured by the $\mathrm{CO}$ or ethane abundance, nor does it seem to be determined mainly by a simple seasonal effect from nucleus shape or distribution of material on the surface. Such seasonal effects when seen seem to be superimposed on the overall variation. Outburst and fragmentation are present in some of these comets across the range of slopes, production rates, and volatility. This clearly tells something about the region of the giant planets where Oort cloud comets formed. Recent evidence from the Stardust mission (Brownlee et al. 2006) indicates that there might have been extensive mixing of high temperature processed refractory material from the inner solar system all the way out to the Kuiper belt. Perhaps the lack of simple correlations indicates that there was also mixing of icy materials condensed in various locations within the early solar system before final cometessimal formation as well.

$\mathrm{SOHO}$ is an international cooperative missions between ESA and NASA. M.R.C. acknowledges support from grant NNG05GF06G from the NASA Planetary Astronomy Program and grant NNX06AB60G from the Outer Planets Research Program. N.J.H. was also partially supported by the NSF REU grant ATM-0552353. J.-L.B. and E.Q. acknowledge support from CNRS and CNES. We thank Dr. H. Boehnhardt for useful discussions regarding split comet $\mathrm{C} / 2001$ A2. We thank Dr. F. Bensch and Dr. A. Lovell both for useful discussions and for providing observational results prior to formal publication. We obtained cometary ephemerides from the JPL Horizons Web site. We also acknowledge the personnel that have been keeping SOHO and SWAN operational for over 10 years.

\section{REFERENCES}

A'Hearn, M. F., Millis, R. L., Schleicher, D. G., Osip, D. J., \& Birch, P. V. 1995, Icarus, 118, 223270

Bensch, F., Bergom, E. A., Bockelée-Morvan, D., Melnick, G. J., \& Biver, N. 2004, ApJ, 609, 1164

Bensch, F., \& Melnick, G. J. 2006, AAS, DPS Meeting \#38, \#33.04

Bertaux, J. L., et al. 1995, Solar Phys., 162, 403

Bertaux, J. L., et al. 1998, Planet. Space Sci., 46, 555

Biver, N., et al. 2000, AJ, 120, 1554

Biver, N., et al. 2006, A\&A, 449, 1255

Bockelée-Morvan, D., Crovisier, J., Mumma, M. J., \& Weaver, H. A. 2004, in Comets II, ed. M. Festou, H. U. Keller, \& H. A. Weaver (Tucson, AZ: Univ. Arizona Press), 391
Boehnhardt, H. 2002, Earth Moon Planets, 89, 91

Bonev, B. P., Mumma, M. J., Dello Russo, N., Gibb, E. L., DiSanti, M. A., \& Magee-Sauer, K. 2004, ApJ, 615, 1048

Brownlee, D., et al. 2006, Science, 314, 1711

Chiu, K., Neufeld, D. A., Bergin, E. A., Melnick, G. J., Patten, B. M., Wang, Z., \& Bockelée-Morvan, D. 2001, Icarus, 154, 345

Combi, M. R., Brown, M. E., Feldman, P. D., Keller, H. U., Meier, R. R., \& Smyth, W. H. 1998, ApJ, 494, 816

Combi, M. R., Mäkinen, J. T. T., Bertaux, J.-L., \& Quémerais, E. 2005, Icarus, 177,228

Combi, M. R., Mäkinen, J. T. T., Henry, N. J., Bertaux, J.-L., \& Quémerais, E 2006, Bull. AAS, 38, 535

Combi, M. R., Reinard, A. A., Bertaux, J.-L., Quémerais, E., \& Mäkinen, T. 2000, Icarus, 144, 191

Combi, M. R., \& Smyth, W. H. 1988a, ApJ, 327, 1026

Combi, M. R., \& Smyth, W. H. 1988b, ApJ, 327, 1044

Dello Russo, N., Bonev, B. P., DiSanti, M. A., Mumma, M. J., Gibb, E. L., Magee-Sauer, K., Barber, R. J., \& Tennyson, J. 2005, ApJ, 621,537

Dello Russo, N., DiSanti, M. A., Magee-Sauer, K., Gibb, E. L., Mumma, M. J., Barber, R. J., \& Tennyson, J. 2004, Icarus, 168, 186

Dello Russo, N., Mumma, M. J., DiSanti, M. A., Magee-Sauer, K., Gibb, E. L., Bonev, B. P., McLean, I. S., \& Xu, L.-H. 2006, Icarus, 184, 2555

Feldman, P. D., Weaver, H. A., A'Hearn, M. F., Festou, M. C., McPhate, J. B., \& Tozzi, G. P. 1999, AAS, DPS Meeting \#31, \#35.02

Furusho, R., Kawakita, H., Jujii, M., Kinugasa, K., Yamamuro, T., \& Takeyama, N. 2003, Publ. Astron. Soc. Japan, 55, 1153

Harris, W. M., Combi, M. R., Honeytcutt, R. K., \& Mueller, B. E. A. 1997, Science, 277, 676

Hatchell, J., Bird, M. K., van der Tak, F. F. S., \& Sherwood, W. A. 2005, A\&A, 439, 777

Helbert, J., Rauer, H., Boice, D. C., \& Huebner, W. F. 2005, A\&A, 442, 1107

Irvine, W. M., Bergman, P., Lowe, T. B., Matthews, H., McGonagle, D., Nummelin, A., \& Owen, T. 2003, Origins Live Evolution Biosphere, 33, 600

Jehin, E., et al. 2002, Earth Moon Planets, 90, 147

Kawakita, H., Watanabe, J., Kinoshita, D., Ishiguro, M., \& Nakamura, R 2003, ApJ, 590, 573

Lara, L.-M., Rodrigo, R., Tozzi, G. P., Boehnhardt, H., \& Leisy, P. 2004a, A\&A, 420,371

Lara, L.-M., Tozzi, G. P., Boehnhardt, H., DiMartino, M., \& Schulz, R. 2004b, A\&A, 422, 717

Lecacheux, A., et al. 2003, A\&A, 402, L55

Lovell, A. J., Howell, E. S., \& Schloerb, F. P. 2008, ApJ, submitted

Magee-Sauer, K., Mumma, M. J., DiSanti, M. A., Dello Russo, N., Gibb, E. L., \& Bonev, B. P. 2008, Icarus, in press

Mäkinen, J. T. T., Bertaux, J.-L., Combi, M. R., \& Quémerais, E. 2001, Science, 292, 1326

Mäkinen, J. T. T., \& Combi, M. R. 2005, Icarus, 177, 217

Mumma, M. J., DiSanti, M. A., Dello Russo, N., Magee-Sauer, K., Gibb, E., \& Novak, R. 2003, Adv. Space Res., 31, 2563-2575

Mumma, M. J., et al. 2001a, ApJ, 546, 1183

Mumma, M. J., et al. 2001b, Science, 292, 1334

Neufeld, D. A., et al. 2000, ApJ, 539, L151

Schleicher, D. G., Millis, R. L., \& Birch, P. V. 1998, Icarus, 132, 397

Sekanina, Z., Jehin, E., Boehnhardt, H., Bonfils, X., \& Schuetz, O. 2002, ApJ, 572,679

Smyth, W. H., Marconi, M. L., \& Combi, M. R. 1995, Icarus, 113, 119

Vervack, R. J. Jr., et al. 2004, AAS, DPS Meeting \#36, \#33.08

Weaver, H., Feldman, P. D., Combi, M. R., Krasnopolsky, V., Lisse, C. M., \& Shemansky, D. E. 2002, ApJ, 576, L95

Weaver, H. A., Davies, J. K., Kerr, T., Vacca, W., Biver, N., Bockelée-Morvan, D., Crovisier, J., Brook, T. Y., Chin, G., \& Kim, S. J. 1999, AAS, DPS Meeting \#31, \#32.05

Weaver, H. A., et al. 2001, Science, 292, 1329 